\title{
Formulating the Kramers problem in field theory
}

\author{
Arjun Berera, ${ }^{1}$ Joël Mabillard, ${ }^{1}$ Bruno W. Mintz $\odot,{ }^{2}$ and Rudnei O. Ramos $\odot^{2}$ \\ ${ }^{1}$ School of Physics and Astronomy, University of Edinburgh, Edinburgh EH9 3FD, United Kingdom \\ ${ }^{2}$ Departamento de Física Teórica, Universidade do Estado do Rio de Janeiro, \\ 20550-013 Rio de Janeiro, RJ, Brazil
}

(Received 3 July 2019; published 10 October 2019)

\begin{abstract}
The escape problem is defined in the context of quantum field theory. The escape rate is explicitly derived for a scalar field governed by fluctuation-dissipation dynamics, through generalizing the standard Kramers problem. In the presence of thermal fluctuations, there is a nonvanishing probability for a classical background field to escape from the well. Different from nucleation or quantum tunneling processes, the escape problem does not require the minimum of the potential, where the field is initially located in a homogeneous configuration, to be a false vacuum. The simple and well-known related problem of the escape of a classical point particle due to random forces is first reviewed. We then discuss the difficulties associated with a well-defined formulation of an escape rate for a scalar field and how these can be overcome. A definition of the Kramers problem for a scalar field and a method to obtain the rate are provided. Finally, we discuss some of the potential applications of our results, which can range from condensed matter systems, i.e., nonrelativistic fields, to applications in high-energy physics, like for cosmological phase transitions.
\end{abstract}

DOI: 10.1103/PhysRevD.100.076005

\section{INTRODUCTION}

The problem of escaping a potential well has been an active field of research over the last century and has applications in several scientific disciplines, such as in physics and chemistry. Classically, a particle put at rest at the bottom of a potential well stays there if left undisturbed. However, in any realistic physical system, we expect the presence of fluctuation and dissipation dynamics, which, for example, naturally emerge through the interactions of the system with a thermal bath. Under these conditions, an escape from the potential well might be allowed. The derivation of the escape rate is called the Kramers problem [1] and is, to a large extent, well understood for the simplest systems, such as a classical point particle. One should note that, in its original zero-dimensional (usual field theory nomenclature for a point particle as a zero space and one time dimensional field) formulation, the escape problem is defined regardless of what is beyond the top of the energy barrier. That is, one is interested in the probability per unit time for the particle to escape the potential well, independently of what happens after this escape. To our knowledge, however, no explicit extension of this problem to a relativistic

Published by the American Physical Society under the terms of the Creative Commons Attribution 4.0 International license. Further distribution of this work must maintain attribution to the author(s) and the published article's title, journal citation, and DOI. Funded by SCOAP. field has been done so far. Such an extension would be very welcome for several possible applications in high-energy physics and cosmology. For example, in the physics of the early Universe, one is interested in describing cosmological fields immersed in a hot medium. Fluctuation-dissipation dynamics has been shown to have interesting applications in the early Universe such as the warm inflation paradigm [2,3] and during a cosmological phase transition [4]. It, therefore, follows to try to define and understand precisely the rate of escape of such fields due to thermal fluctuations.

Computing the probability for a classical particle to diffuse has always been of great interest, in particular in the context of stochastic dynamics. Several methods have been proposed over the years. Kramers, a pioneer in the field, derived the so-called Kramers rate [1] using the flux-overpopulation method based on ideas originally developed by Farkas in Ref. [5]. Another way to obtain the escape rate is achieved with the mean-first-passage-time (MFPT) formalism using the adjoint Fokker-Planck (FP) operator [6]. However, this approach is more delicate to handle due to complex boundary conditions. A third method consists of finding the smallest positive, nonvanishing eigenvalue of the FP operator. It has been shown that this eigenvalue is directly related to the escape rate [7]. A comprehensive review of these methods can be found in [8]. More recently, in [9], it was shown that there is a universal equivalence between these different approaches.

When regarding a field instead of a particle, the situation changes significantly. A somewhat related problem in 
quantum physics is that of the study of quantum tunneling. The decay rate of a field has been derived by Callan and Coleman at zero temperature $[10,11]$ and extended to finite temperature by Linde [12] (also known as the nucleation problem in finite temperature quantum field theory [13]). The inclusion of gravitational effects has been studied by Coleman and de Luccia in Ref. [14]. The formalism describing a field subject to random forces is a developed topic called stochastic field theory $[15,16]$, but in spite of this there has never been a precise and complete discussion of the escape problem. One of the main difficulties is the identification of the most suitable approach to be generalized to a scalar field. Zinn-Justin in Ref. [16] briefly states the problem and suggests deriving the smallest eigenvalue and the use of instantons. This is indeed a possibility but, unfortunately, it faces some analytical limitations when deriving the rate. We find that the work of Langer $[17,18]$ in extending the flux-over-population method to a $2 \mathrm{~N}$-dimensional system appears as the most promising approach to be used with a field.

The field theory aspect of the problem renders the definition of an escape more difficult and less intuitive than for a single point particle. In particular, the actual shape of the potential beyond the potential well plays a role in the computation of the rate for the field. However, as in the zero-dimensional case, the Kramers problem can be defined for both an initial true or false vacuum. Using the ideas and the formalism of the flux-over-population method extended to a field, we will propose in this work a definition of the Kramers problem and explicitly evaluate the rate. Along the way in this derivation, we will encounter some familiar situations, such as the Hawking and Moss solution [19]. We will also compare our final result for the escape rate with the known result of nucleation rate due to thermal fluctuations [12,13]. In particular, considering the wellknown result of Linde for the quantum tunneling rate at finite temperature [12], we will show that, in the limit where the temperature is sufficiently high for the thermal fluctuations to dominate over the quantum fluctuations, the nucleation rate is proportional to the escape rate. This is remarkable since the two results are based on completely different approaches. This result shows that, when the system is initially in a false vacuum, the nucleation rate is indeed a special case of the escape rate.

Apart from the formal interest in the computation of an escape rate for a scalar field, the result has potentially many applications. The process helps to give a thorough understanding of out-of-equilibrium situations, for example during phase transitions. In particular, this process can influence the formation of topological defects and potentially alter the stability of the embedded configurations. In addition, the escape rate is a well-suited mechanism for situations where the field needs to probe several local minima. Such a situation appears in string theory, with the string landscape, and, also, in condensed matter physics, in the context of the glass transition, just to cite some of the potential applications. Another interesting application can be to stochastic inflation. Moreover, our derivation is formally identical to the stochastic quantization, used in particular in lattice field theory, where the origin of the stochastic forces is quantum instead of thermal. A precise knowledge of a transition rate is therefore of great interest in this context.

The aim of this paper is, therefore, to formulate a known problem, the definition and the derivation of the Kramers escape rate, to a physical situation where it has not been applied yet, a scalar field theory. For this, we first need to define the escape problem consistently in the context of field theory. We then will obtain an explicit expression for the escape rate in field theory. For our derivation we have identified an already known method, the flux-over-population method $[1,17,18]$, which we show can be generalized to attain the result we seek. The main result for the escape rate in field theory is Eq. (3.69). Unsurprisingly, it has many similarities to the expression for the particle case, but there are also distinct differences, as will be evident from our derivation and summarized below this main result. Once a general expression for the rate is obtained, we also discuss some techniques, such as the thin-wall approximation, in order to obtain an analytical expression for the rate, when a potential is specified. Beyond the actual result, the method itself suggests an approach for several applications which is, after all, as essential as the actual result for the rate.

The present paper is organized as follows. Section II gives a brief review of the Kramers problem and the methods necessary to compute the escape rate in the simplest case of a point particle. We will focus on one of the main approaches for the computation of the Kramers rate, the flux-over-population method, which is the best candidate to be generalized to a scalar field. Another approach, the MFPT, not used here, is however discussed in the Appendix. The MFPT provides a simple interpretation of the escape rate as we will see. We also present the proof of the equivalence of the two methods. Section III first states the difficulties in the formulation of an escape problem for a scalar field. We then review some basics of stochastic field theory with the Langevin and the associated FP equation. We then define and derive the escape rate for a scalar field using the flux-over-population method. This is the main result of this work. In Sec. IV we compare our result for the escape rate with a closely related problem, that of quantum tunneling dominated by thermal fluctuations, and outline some of the similarities and fundamental differences between the two cases. Section V discusses some potential applications for our results. Finally, Sec. VI has some concluding remarks.

\section{RATE OF ESCAPE OF A CLASSICAL POINT PARTICLE}

To introduce the escape problem and the associated computations, we consider the simplest and well-known 
example of a classical point particle in a metastable potential, whose dynamics is subject to both a dissipative and a stochastic force. The two equivalent formalisms, based on the Langevin and the FP equations, are reviewed. We then also review the flux-over-population and the mean-first-passage-time methods, which are used to obtain the escape rate, that is, the probability per unit time that the particle crosses the top of the potential energy barrier (and, therefore, escapes the metastable minimum).

\section{A. Point particle in a metastable potential}

We consider a classical point particle of mass $m$ initially located at a local minimum $x_{A}$ of the potential $V(x)$. For simplicity, we assume only one direction of escape, which may happen through the closest local maximum located at $x_{B}$ on the right of $x_{A}$. On the left of the local minimum, the potential is assumed to rise and not to have any additional extrema. The situation is depicted in Fig. 1. Beyond the local maximum at $x_{B}$, to the right the potential might have another local or global minimum or be unbounded from below; it does not matter. The height of the barrier is $\Delta V$. In particular, the escape rate should be independent of the shape of the potential beyond the top of the barrier to the right of $x_{B}$.

In a classical deterministic description, the particle sitting at the local minimum stays there forever and an escape from the potential well is not allowed. Its dynamics is governed by Newton's second law,

$$
m \frac{d^{2} x}{d t^{2}}=-V^{\prime}(x)
$$

where the prime denotes a derivative with respect to $x$. The position $x_{A}$ at the local minimum of the potential is stable. In other words, $x_{A}$ is an attractor. Under a small perturbation, the particle comes back to the original position.

In the presence of a thermal bath or a fluid, in which the particle is placed, the situation is altered by the two competing effects intrinsic to fluctuation and dissipation

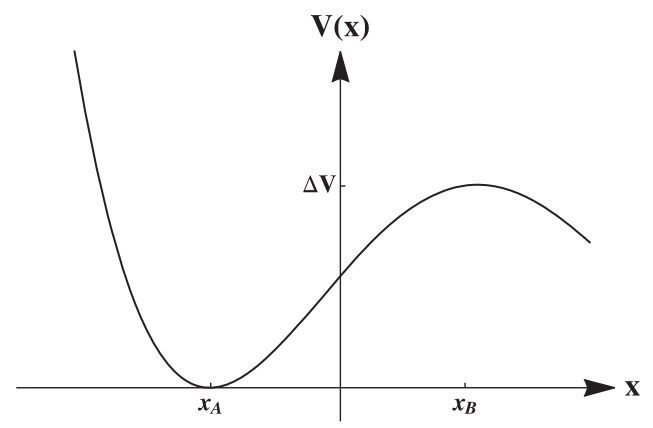

FIG. 1. Potential corresponding to the escape problem. The position $x_{A}$ is the local minimum, where the point particle sits initially, and $x_{B}$ the local maximum of the potential. The barrier height is denoted by $\Delta V$. dynamics. The random forces, originating from the thermal fluctuations, push the particle away from the initial position and allow it to eventually climb the potential barrier. In addition, the damping tends to slow down the particle and makes the return to the equilibrium point $x_{A}$ more difficult. Due to the combined effect of fluctuation and dissipation, the system is not stable anymore and there is a nonzero probability for the particle to escape from the well. In particular, after a sufficiently long time, it is reasonable to expect that the particle has almost surely (i.e., it has a nonvanishing probability to have) passed over the barrier.

We are interested in the rate at which the particle escapes from the potential well. The escape rate is closely related to the inverse of the average time needed to pass, for the first time, the local maximum of the potential. This time is known in the literature as the "mean first passage time" [6]. A naive inspection indicates that the escape rate should only depend on the damping coefficient, the strength of the noise, the temperature, and the potential. In regards to the latter, in particular, the rate depends on the height of the barrier and the curvature at the minimum and at the maximum. Since the escape is defined from the first passage at the top of the barrier, the characteristics of the potential beyond the maximum should not play any role.

One clarification on terminology is worth stating here. For a classical point particle, the escape rate is different from and should not be confused with a diffusion rate to the next minimum. The diffusion rate is typically smaller than the escape rate since, once the particle has passed over the top, it must then go down the potential on the other side and, eventually, reach the minimum. If the next minimum is at lower energy, the diffusion rate is a decay rate. Let us now formulate the escape problem.

\section{B. Langevin and Fokker-Planck descriptions}

The Langevin and the FP formalisms are two equivalent approaches used to describe a particle subject to random forces that follow a Markov process. Both approaches are presented here with a discussion of their strengths and limitations.

\section{The Langevin equation}

The Langevin equation is obtained by the inclusion of the random force, parametrized with a stochastic noise $\xi(t)$, and the damping term, all in the form of Newton's second law,

$$
m \frac{d^{2} x}{d t^{2}}=-\eta \frac{d x}{d t}-V^{\prime}(x)+\xi(t)
$$

where $\eta$ is the damping coefficient. For simplicity, the noise will always be assumed to be Gaussian throughout this work. The average over the noise of an operator $\mathcal{O}(x)$ is defined as 


$$
\langle\mathcal{O}(x)\rangle_{\xi} \equiv \int d[\xi] \mathcal{O}\left(x_{\xi}\right) \exp \left\{-\frac{1}{2 \Omega} \int_{t_{0}}^{t_{f}} d t^{\prime} \xi^{2}\left(t^{\prime}\right)\right\}
$$

where $x_{\xi}$ is the solution of the Langevin equation (2.2) for a given realization $\xi$ of the noise. Here, $t_{0}$ and $t_{f}$ are the initial and final times. The noise here satisfies the following relations:

$$
\langle\xi(t)\rangle_{\xi}=0, \quad\left\langle\xi(t) \xi\left(t^{\prime}\right)\right\rangle_{\xi}=\Omega \delta\left(t-t^{\prime}\right),
$$

where $\Omega$ parametrizes the strength of the noise. The damping coefficient $\eta$ is related to $\Omega$ by the Einstein relation $\Omega=2 \eta k_{B} T$.

The Langevin equation is a stochastic differential equation for the random variable $x_{\xi}$ and is, therefore, not deterministic. Given that the randomness of the stochastic force actually follows a well-defined probability distribution (in our case, a Gaussian white noise Markovian process), the random variable $x_{\xi}$ should also obey some probability distribution $\rho(x)$. The FP equation is the equation whose solution is precisely this probability distribution $\rho(x)$. In practice, given that it is a partial differential equation with well-defined coefficients and boundary conditions, the FP equation is better suited for an analytical treatment than the Langevin equation.

\section{The Fokker-Planck equation}

The idea behind the FP description is to consider the evolution of the probability distribution of the quantities of interest, in our case, the position and the velocity of the particle. Due to the presence of random forces, each realization is achieved with a certain probability. Even though each individual particle dynamics realization is nondeterministic, the evolution of the probability distribution is deterministic.

We are interested in the position and the velocity of the particle as a function of time. The Langevin equation gives a set of two first-order differential equations for the position $x(t)$ and velocity $v(t)$,

$$
\begin{aligned}
\frac{d x}{d t} & =v, \\
m \frac{d v}{d t} & =-\eta v-V^{\prime}(x)+\xi(t) .
\end{aligned}
$$

The FP probability distribution is defined as

$$
P\left(x, v, t \mid x_{0}, v_{0}, t_{0}\right) \equiv\left\langle\delta\left[x_{\xi}(t)-x\right] \delta\left[v_{\xi}(t)-v\right]\right\rangle_{\xi},
$$

where the arguments $x_{\xi}(t)$ and $v_{\xi}(t)$ of the Dirac deltafunctions on the right are the solutions of the Langevin equation [in the presence of the random force $\xi(t)$ ] and $x$ and $v$ the arguments of the probability distribution. $P$ is the averaged probability to find the particle at position $x$ with velocity $v$ at time $t$, knowing the initial position $x_{0}$ and velocity $v_{0}$ at time $t_{0}$.

The probability distribution satisfies the FP equation ${ }^{1}$

$\frac{\partial}{\partial t} P\left(x, v, t \mid x_{0}, v_{0}, t_{0}\right)=-\mathcal{L}_{\mathrm{FP}} P\left(x, v, t \mid x_{0}, v_{0}, t_{0}\right)$,

where $\mathcal{L}_{\mathrm{FP}}$ is the FP operator defined as

$$
\mathcal{L}_{\mathrm{FP}} \equiv \frac{\partial}{\partial x} v-\frac{1}{m} \frac{\partial}{\partial v}\left[\eta v+V^{\prime}(x)\right]-\frac{\Omega}{2 m^{2}} \frac{\partial^{2}}{\partial v^{2}} .
$$

The FP equation is an ordinary differential equation for the probability distribution $P$ and, therefore, analytical methods can be applied.

In the large time limit, the system is expected to reach equilibrium. The equilibrium probability distribution $P_{0}$ is a time-independent solution of the FP equation given by

$$
\begin{aligned}
P_{0}(x, v) & =\frac{1}{\mathcal{Z}} \exp \left\{-\beta\left(\frac{1}{2} m v^{2}+V(x)\right)\right\} \\
& =\frac{1}{\mathcal{Z}} \exp \{-\beta E(x, v)\},
\end{aligned}
$$

where $E$ is the energy of the nondissipative system and the partition function $\mathcal{Z}$ is the normalization. Note that the equilibrium distribution always formally exists as a solution of the FP equation; however, it does not necessarily imply that the system possesses an equilibrium state. The equilibrium distribution can be non-normalizable, in particular, if the potential is unbounded from below. The FP formalism is fully equivalent to the Langevin approach and provides the tools needed for an analytical derivation of the escape rate.

\section{Computation of the escape rate}

Over the last century, several methods have been proposed to estimate the escape rate. ${ }^{2}$ Since our final goal is to consider a relativistic scalar field, we focus on the flux-overpopulation method that appears as the most promising candidate for such a generalization. For a better interpretation of the escape problem, we introduce, in the Appendix, the framework of the MFPT and show its formal equivalence with the flux-over-population method, which proves that the escape rate is indeed the inverse of the MFPT.

\section{Flux-over-population method}

The flux-over-population method has been introduced in Ref. [5] and the explicit computation of the rate has been achieved by Kramers in Ref. [1].

\footnotetext{
${ }^{1}$ Explicit details on the derivation and a discussion about the properties of this equation can be found in Refs. [7,16].

${ }^{2}$ For a comprehensive review of these methods, see, e.g., Ref. [8].
} 




FIG. 2. Example case studied with the flux-over-population method. The blue solid line is the potential $V(x)$. The red dasheddotted line is the particle's initial position and the green dotted line is the equilibrium FP probability distribution for the position. The position $x_{A}$ is the initial location of the particle, $x_{B}$ the local maximum, $x_{C}$ a second local minimum, $x_{S o}$ and $x_{S i}$ the positions of the source and the sink respectively.

Let us consider the situation shown in Fig. 2. For illustrative purposes, we have chosen an asymmetric double-well potential. Similar reasoning applies to any kind of potential as long as it possesses a local minimum in the vicinity of a local maximum. ${ }^{3}$ The particle is initially located at the minimum $x_{A}$ and the FP probability distribution at time $t_{0}$ is a product of two Dirac delta-functions,

$$
P\left(x, v, t_{0} \mid x_{A}, 0, t_{0}\right)=\delta\left(x-x_{A}\right) \delta(v) .
$$

The position-dependent parts of the initial and equilibrium probability distributions are shown in Fig. 2, with the red dashed-dotted line and the green dotted line, respectively. During the time evolution, given by the FP equation (2.8), the probability distribution goes from the red dashed-dotted line to the green dotted line. In the large time limit, the system has reached equilibrium and the probability distribution is given by Eq. (2.10). Therefore, there must be a flux of probability at the maximum of the well. The origin of this flux of probability is precisely the fluctuation and dissipation dynamics discussed previously.

The idea behind the flux-over-population method relies on the construction of a steady-state solution. The inclusion of sources and sinks maintains a constant probability current across the well. The role of the sources, located to the left of the minimum at $x_{S o}$ in Fig. 2, is to supply particles to the " $A$-well" and maintain a constant number density inside the well. The particles thermalize and eventually leave the well before being removed by the sinks, located on the right of the maximum at $x_{S i}$. Since the total probability flux $j$ is equal to the rate of escape $k$ times the population of the $A$-well, $n_{A}$, the flux-over-population method predicts

\footnotetext{
${ }^{3}$ The shape of the potential influences the form of the equilibrium distribution; however, the existence of a probability flux at the top of the potential is guaranteed.
}

$$
k \equiv \frac{j}{n_{A}}
$$

as a solution for the escape rate.

The population of the $A$-well is given by the integration over the probability density,

$$
n_{A}=\int_{A-\text { well }} d x d v P(x, v),
$$

which corresponds to the probability to be in the well, with $x \in\left(-\infty, x_{B}\right]$ and $v \in(-\infty,+\infty)$. The flux at the barrier is

$$
j=\int_{-\infty}^{+\infty} d v v P\left(x_{B}, v\right)
$$

which is the probability to pass over the maximum with some velocity.

The derivation of the rate requires two steps. First, the probability distribution is obtained and then second the flux and the number density are computed. The probability density $P$ is a solution of the FP equation (2.8) with the particular boundary conditions dictated by the specific steady-state situation under consideration. The ensemble of particles is in equilibrium inside the $A$-well and the probability density is given by Eq. (2.10). Since the sinks remove the particles once they have passed the maximum, we impose

$$
P\left(x>x_{S i}, v\right) \simeq 0 .
$$

Finally, at the top of the barrier, there are no sources or sinks and the potential $V(x)$ is approximated as

$V(x) \simeq V\left(x_{B}\right)-\frac{1}{2}\left|V^{\prime \prime}\left(x_{B}\right)\right|\left(x-x_{B}\right)^{2}+\mathcal{O}\left[\left(x-x_{B}\right)^{3}\right]$,

and, therefore, the steady-state FP equation (2.8) becomes

$$
\begin{gathered}
\left\{-\frac{\partial}{\partial x} v+\frac{1}{m} \frac{\partial}{\partial v}\left[\eta v-\left|V^{\prime \prime}\left(x_{B}\right)\right|\left(x-x_{B}\right)\right]\right. \\
\left.+\frac{\Omega}{2 m^{2}} \frac{\partial^{2}}{\partial v^{2}}\right\} P(x, v)=0
\end{gathered}
$$

at the vicinity of the top of the barrier $x_{B}$.

The construction of $P(x, v)$ relies on the Kramers ansatz [1],

$$
P(x, v)=\zeta(x, v) P_{0}(x, v),
$$

where $P_{0}$ is the equilibrium distribution and $\zeta$ is chosen to satisfy the boundary conditions:

$$
\lim _{x \rightarrow x_{A}} \zeta(x, v)=1, \quad \zeta\left(x>x_{S i}, v\right)=0 .
$$


Applying the FP operator on the ansatz and using the equilibrium distribution Eq. (2.10), we obtain the equation for $\zeta$,

$$
\begin{gathered}
\left\{-v \frac{\partial}{\partial x}-\frac{1}{m}\left[\eta v+\left|V^{\prime \prime}\left(x_{B}\right)\right|\left(x-x_{B}\right)\right] \frac{\partial}{\partial v}\right. \\
\left.+\frac{\Omega}{2 m^{2}} \frac{\partial^{2}}{\partial v^{2}}\right\} \zeta(x, v)=0,
\end{gathered}
$$

where we identify the adjoint FP equation

$$
\mathcal{L}_{\mathrm{FP}}^{\dagger} \zeta(x, v)=0 .
$$

In order to solve this equation, Kramers made the further assumption in [1] that $\zeta$ depends only on $u$, a linear combination of $x$ and $v$, such that

$$
u \equiv\left(x-x_{B}\right)+a v .
$$

From a purely mathematical point of view, this assumption allows finding a solution of the differential equation (2.20) that satisfies the boundary conditions (2.19). According to Ref. [18], it can be shown that the form of solution $\zeta(u)$ is unique.

A physical interpretation for adopting the ansatz (2.20) can be obtained by looking at the function $\zeta(x, v)$. From its definition (2.18), $\zeta$ parametrizes the deviation from equilibrium due to thermal activation in the vicinity of the saddle point. The second boundary condition in (2.19) implies that $\zeta(x, v)$ should go to zero in the region of phase space away from the saddle point (the "probability sink" at $x \gg x_{B}$ ) and also should quickly vanish in the region of positive velocities. Away from the saddle point, it is fair to expect that the vanishing of the function $\zeta(x, v)$ is controlled by either $\left(x-x_{B}\right)$ or $v$. The linear combination in the Kramers assumption (2.22) is the simplest and most straightforward way to implement this idea. The equation for $\zeta(u)$ then becomes

$-\left[\left(1+\frac{a}{m} \eta\right) v+\frac{a}{m}\left|V^{\prime \prime}\left(x_{B}\right)\right|\left(x-x_{B}\right)\right] \zeta^{\prime}+a^{2} \frac{\Omega}{2 m^{2}} \zeta^{\prime \prime}=0$,

where the prime denotes a $u$-derivative. For consistency with the assumption that $\zeta$ is a function of $u$ only and in order to obtain the correct behavior at the boundary, the factor in front of $\zeta^{\prime}$ must be a linear function of $u$. Imposing that

$$
\lambda u \equiv-\left[\left(1+\frac{a}{m} \eta\right) v+\frac{a}{m}\left|V^{\prime \prime}\left(x_{B}\right)\right|\left(x-x_{B}\right)\right],
$$

the constants $a$ and $\lambda$ are found to be given, respectively, by

$$
\begin{aligned}
& \lambda_{ \pm}=-\frac{\eta}{2 m} \pm \sqrt{\frac{\left|V^{\prime \prime}\left(x_{B}\right)\right|}{m}+\left(\frac{\eta}{2 m}\right)^{2}}, \\
& a=-\frac{m}{V^{\prime \prime}\left(x_{B}\right)} \lambda_{ \pm},
\end{aligned}
$$

where the two solutions for $\lambda$ have opposite signs.

Solving for $\zeta(u)$ by inserting Eq. (2.24) in the differential equation and integrating twice gives

$\zeta(u)=\sqrt{\frac{\beta\left[V^{\prime \prime}\left(x_{B}\right)\right]^{2}}{2 \pi \eta \lambda_{+}}} \int_{u}^{\infty} d z \exp \left\{-\beta \frac{\left[V^{\prime \prime}\left(x_{B}\right)\right]^{2}}{2 \eta \lambda_{+}} z^{2}\right\}$,

where $\lambda_{+}$has been chosen to have an overall negative exponent and, therefore, $\zeta$ to vanish for large positive $x$. The factor in front of $\zeta$ has been chosen to satisfy the condition that $\zeta$ goes to unity inside the $A$-well.

Having all elements at our disposal to compute the probability flux $j$, we obtain the result

$$
j=\frac{1}{\mathcal{Z}}\left(\frac{\lambda_{+}}{\beta}\right) \frac{1}{\sqrt{m\left|V^{\prime \prime}\left(x_{B}\right)\right|}} \exp \left\{-\beta V\left(x_{B}\right)\right\},
$$

where we have used integration by parts. The population $n_{A}$ of the $A$-well is simply

$$
n_{A} \simeq \frac{1}{\mathcal{Z}} \sqrt{\frac{2 \pi}{\beta m}} \sqrt{\frac{2 \pi}{\beta V^{\prime \prime}\left(x_{A}\right)}} \exp \left\{-\beta V\left(x_{A}\right)\right\},
$$

where the potential has been expanded around the local minimum in $x_{A}$ and the limit of integration for $x$ safely extended to infinity.

Taking the ratio of $j$ and $n_{A}$, the escape rate is found to be

$$
\begin{aligned}
k= & \frac{\sqrt{\frac{\left|V^{\prime \prime}\left(x_{B}\right)\right|}{m}+\left(\frac{\eta}{2 m}\right)^{2}}-\frac{\eta}{2 m}}{2 \pi} \sqrt{\frac{V^{\prime \prime}\left(x_{A}\right)}{\left|V^{\prime \prime}\left(x_{B}\right)\right|}} \\
& \times \exp \left\{-\beta\left[V\left(x_{B}\right)-V\left(x_{A}\right)\right]\right\},
\end{aligned}
$$

which is the famous result of Kramers. As expected, the rate depends only on the parameters $\eta$ (or equivalently $\Omega$ ), the temperature, the curvature of the potential at the initial local minimum and the nearby maximum and the height of the barrier. The height of the barrier $\Delta V=V\left(x_{B}\right)-V\left(x_{A}\right)$ can be seen as the activation energy.

It is important to notice that the shape of the potential on the other side of the well does not influence the final result. This is a consequence of the absorbing boundary condition (2.15). Notice that this feature is crucial for a sound definition of the escape problem. Indeed, for a potential 
well such as the one shown in Fig. 2, there is a minimum at $x_{C}$, which has a higher potential energy compared to the starting well at $x_{A}$. In this case, the total probability flow over the barrier at $x_{B}$ will be made up of a sum of two contributions: one escaping contribution (from $x_{A}$ to $x_{C}$ ) and a returning contribution (from $x_{C}$ back to $x_{A}$ ). The absorbing boundary condition (2.15) is a way to disentangle the two contributions, keeping only the escaping part of the probability flux over the barrier.

An alternative method to derive the escape rate is achieved with the formalism of the mean first passage time. This approach provides a simple interpretation of the escape problem; it corresponds to the average time needed to leave for the first time a specified domain. In practice, it is difficult to solve for the MFPT; this is due, in particular, to nontrivial boundary conditions. In the Appendix, we provide more details on the formalism of the MFPT and show the formal equivalence with the flux-over-population method.

\section{ESCAPE RATE FOR A SCALAR FIELD}

The main objective of this work is the definition of the Kramers problem in field theory. Using the knowledge gathered from the classical point particle case, we first describe the escape problem for a scalar field and then show that the formulation of a meaningful definition is not straightforward.

We introduce the two usual formulations for dealing with the Kramers problem. The first is based on the Langevin equation, which has a direct interpretation but is limited in its analytical treatment. The second formulation is the one based on the FP approach, whose derivation is more involved, but is much more amenable to analytic treatment. In this section we will also use ideas from the flux-overpopulation method to define the Kramers problem, derive explicitly the escape rate for a scalar field and then interpret the results.

\section{A. Defining the escape problem for a background scalar field configuration}

Let us consider a scalar field with a self-interaction potential as shown in Fig. 3. We assume, for simplicity, that the initial configuration is a homogeneous field sitting at a local minimum $\phi^{A}$. The interactions with extra degrees of freedom (d.o.f.), for example a thermal bath, lead to fluctuation and dissipation dynamics and potentially allow for an escape from the potential well. Our goal is to compute the rate per unit volume for the field to escape from the well, due to thermal fluctuations.

Involving a field renders the definition of an escape more difficult and less intuitive than treating the point particle, zero-dimensional case, discussed previously. At equilibrium, the field populates both sides of the well (or has completely decayed if the potential is unbounded from

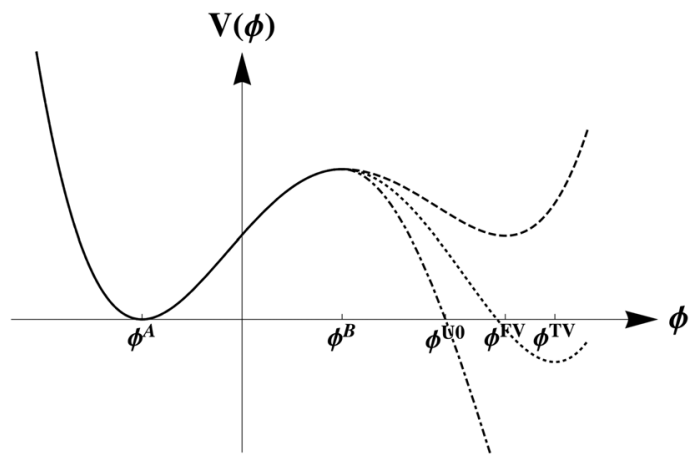

FIG. 3. Potentials corresponding to the escape problem. $\phi^{A}$ is the initial local minimum. $\phi^{B}$ corresponds to the local maximum of the potential. On the right-hand side of the maximum, there are several possibilities, a false vacuum at $\phi^{\mathrm{FV}}$ (dashed line), a true vacuum at $\phi^{\mathrm{TV}}$ (dotted line) or a potential that is unbounded from below (dashed-dotted line) with $V\left(\phi^{U 0}\right)=V\left(\phi^{A}\right)$.

below beyond the well). Comparing with the initial situation, where the field configuration is homogeneously located at $\phi^{A}$, it is reasonable to assume the existence of a flow of the probability density across the potential well. For this reason, the flux-over-population method should apply. The naive generalization of the point particle case would be to estimate the average time needed for the field to reach the top of the potential $\phi^{B}$ for the first time at each point in space. As we will learn, this case can be related to the Hawking-Moss solution [19] in the early Universe. However, in our case, where we are considering a Minkowski spacetime and the volume can be infinite, this solution would lead to a vanishing rate. We should, therefore, seek for another definition of the escape configuration.

Before going into the details of our calculations, it is important to comment on the difference between the escape problem treated in this work with two other closely related problems, the quantum tunneling and the nucleation problem. Quantum tunneling, as its name implies, is a consequence of the quantum fluctuations of the field. Such fluctuations can connect two classically disconnected values of the field, through a forbidden region in potential energy, without giving the field any energy. This is what happens in a quantum first-order phase transition. Nucleation, on the other hand, is the mechanism that drives first-order phase transitions with small degrees of metastability (for example, small supercooling). It corresponds to the formation (or "nucleation") of bubbles of the stable phase inside the metastable phase. Such bubbles grow and complete the phase conversion. Differently from tunneling, the process of nucleation is typically driven by thermal fluctuations (even though for many systems quantum fluctuations may also play a role). In this sense, it can be said that, in nucleation, the potential energy barrier is overcome with energy absorbed from the heat reservoir, in contrast to tunneling. Lastly, the problem treated in this work, the escape problem, does not necessarily require the 
presence of an initial false vacuum. If it is the case, the escape problem can be seen as the first stage of the nucleation problem, i.e., the generation of domains of field configurations outside the initial minimum. In general, the Kramers problem for a scalar field, defined in this work, should be understood as the derivation of the probability for the field to pass over the potential barrier in a finite region of space. Due to thermal fluctuations (quantum fluctuations could also be eventually be considered in a future work), a field starting at a low minimum of potential energy can gain energy from the heat reservoir and then "climb" the potential well to reach and surmount an energy barrier. Another distinctive feature of the escape problem is that it does not regard the dynamics of the field after it finds itself outside of the starting well. However, this issue is more subtle for a field than for a point particle system, as we will discuss below.

As stated in Ref. [16], it is sufficient that a finite part of space has passed the barrier. At first sight, this statement would give some freedom in the precise definition of the escape problem. In particular, once the field has reached the top at a spatial location, it can fall on the other side and attract the neighboring points, with a gain in potential energy, likely accompanied by an energy cost due to inhomogeneities, i.e., a nonzero gradient term. This is a crucial difference with the zero-dimensional, one-particle case of the previous section. When considering a field, the form of the potential beyond the maximum plays a role in the escape problem. Indeed, the Laplacian term in the field equation of motion connects neighboring points of space, which tend to have close values of the field and are thus not independent of each other. Therefore, if a given point of space acquires a field value beyond the maximum of the potential, this is in a way of saying it "communicated" to its neighboring points. This is a distinctive trait of the field system's dynamics that differs from the single particle case. It is then fair to expect that the two cases, where the initial minimum is a true or a false vacuum, must be treated separately. As we will learn shortly, these features naturally emerge along the computation in a generalized flux-overpopulation method and this approach allows for a satisfactory definition of the escape problem. In particular, a critical volume of space that experiences hopping is precisely defined by the formalism. To perform this analysis, we should first introduce some of the necessary ingredients from stochastic field theory.

\section{B. Stochastic field theory}

Let us review here some of the basics of stochastic field theory and introduce the relevant quantities needed for the derivation of the escape rate.

\section{The Langevin and Fokker-Planck equations}

The dynamics of a classical field configuration under random fluctuations and dissipation is an extremely important subject in many different branches in modern physics as far as the description of nonequilibrium fields is concerned (for a thorough introduction and review, see, e.g., Ref. [20]). A natural characteristic when studying the evolution of a system in interaction with an environment is the presence of both dissipative and stochastic terms. For instance, in the context of quantum fields, we might be interested in the derivation of an effective equation of motion for a given field background configuration which represents some relevant characteristic of the system under study (e.g., a vacuum expectation value taking the role of an order parameter important in a phase transition problem). Typically, this involves a selection of a relevant field mode, in which we are interested in the dynamics and will represent the physical system, while the remaining d.o.f. are taken as an environment. The d.o.f. that are regarded as environment can also include any other fields in the original model Lagrangian (see for instance Ref. [20] for a review and discussion about these types of equations and their derivation in the context of quantum field theory). In quantum field theory, the preferred methodology used to study dynamical effects in general is the closed time path formalism [21]. The effective equation of motion for an interacting scalar field is Langevin-like and includes an explicit fluctuation-dissipation relation (see, e.g., Refs. [22-26]). Generically, the usual relativistic Klein-Gordon equation describing the dynamics of the scalar field in a potential $V(\phi)$ is modified to take the thermal fluctuations into account and becomes a Langevin equation,

$$
\left(\partial_{t}^{2}-\nabla^{2}\right) \phi(\vec{x}, t)+\frac{\partial V(\phi)}{\partial \phi}+\eta \dot{\phi}(\vec{x}, t)=\xi(\vec{x}, t),
$$

where $\eta$ is the dissipation coefficient and $\xi$ is a Gaussian white noise satisfying

$$
\begin{aligned}
\langle\xi(\vec{x}, t)\rangle & =0, \\
\left\langle\xi(\vec{x}, t) \xi\left(\vec{x}^{\prime}, t^{\prime}\right)\right\rangle & =\Omega \delta^{3}\left(\vec{x}-\vec{x}^{\prime}\right) \delta\left(t-t^{\prime}\right),
\end{aligned}
$$

where $\Omega$ parametrizes the strength of the noise and satisfies the Einstein relation $\Omega=2 \eta / \beta=2 \eta k_{B} T$. In the quantum field theory context, both the potential and the dissipation coefficient in Eq. (3.1) can be functions of the temperature and on the details of the interactions in the full original microscopic Lagrangian density, carrying, for example, information on the interactions of the scalar field $\phi$ with other field d.o.f. In the following, we will assume a particular fixed form for the potential and the dissipation coefficient. It is straightforward to generalize the analysis for other forms, for example, that include the dependence on the temperature. Exploring the full quantum origin of the Langevin equation for the expectation value of a field goes beyond the scope of this work. We refer the interested reader to [20] and references therein. Thus, for the rest of this work, we will 
simply assume the existence of a Langevin equation of the form of Eq. (3.1).

As an important point of detail, when dealing with integrals over the field space, we discretize the space by adopting the following conventions:

$$
\begin{aligned}
\phi(\vec{x}, t) & \rightarrow \phi\left(x_{i}, t\right)=\phi_{i}(t), \\
\int d^{3} \vec{x} & \rightarrow a^{3} \sum_{i=1}^{N^{3}}, \\
\delta(\vec{x}-\vec{y}) & \rightarrow \frac{\delta_{i j}}{a^{3}},
\end{aligned}
$$

such that

$$
\int d^{3} \vec{x} \delta(\vec{x}-\vec{y})=a^{3} \sum_{i=1}^{N^{3}} \frac{\delta_{i j}}{a^{3}}=1,
$$

where the volume $\mathcal{V}=L^{3}=(N \cdot a)^{3}$, with $N$ being the number of discrete sites in each direction and $a$ the spacing between two adjacent points. Taking into account the field and its conjugate momentum, in a space of three dimensions, we are considering a $2 N^{3}$-dimensional system. For simplicity, we have labeled the spatial points in the three spatial directions with a single label ${ }_{i}$ instead of ${ }_{i j k}$. For the sake of clarity, we will also denote the Laplacian as $\nabla_{i j}^{2} \phi_{j}$. The actual definition in discrete space is given by

$$
\begin{aligned}
\nabla^{2} \phi_{i j k}= & \frac{1}{a^{2}}\left[\phi_{i+1, j, k}+\phi_{i-1, j, k}+\phi_{i, j+1, k}\right. \\
& \left.+\phi_{i, j-1, k}+\phi_{i, j, k+1}+\phi_{i, j, k-1}-6 \phi_{i, j, k}\right]
\end{aligned}
$$

where each direction of space has been explicitly labeled.

As usual when working with the Langevin equation like in Eq. (3.1), the analytical treatment is limited by the stochastic character of the equation. There is, therefore, a need to introduce the FP formalism for the scalar field. The Langevin equation (3.1) implies the following set of equations for the field $\phi$ and the conjugate momentum $\pi$ :

$$
\begin{aligned}
& \partial_{t} \phi_{i}(t)=\pi_{i}(t), \\
& \partial_{t} \pi_{i}(t)=-\eta \pi_{i}(t)+\nabla_{i j}^{2} \phi_{j}(t)-V^{\prime}\left(\phi_{i}\right)+\xi_{i}(t),
\end{aligned}
$$

where the prime denotes a derivative with respect to the field. The FP probability density is defined as

$P\left(\phi, \pi, t \mid \phi_{0}, \pi_{0}, t_{0}\right) \equiv\left\langle\prod_{i=1}^{N^{3}} \delta\left[\hat{\pi}_{i}(t)-\pi_{i}\right] \cdot \delta\left[\hat{\phi}_{i}(t)-\phi_{i}\right]\right\rangle_{\xi}$,

where $\hat{\phi}_{i}(t)$ and $\hat{\pi}_{i}(t)$ are solutions of the Langevin equation (3.6) for a given noise realization $\xi$ and $\phi_{i}$ and $\pi_{i}$ are the arguments of the probability distribution $P$. The stochastic average of an operator $\mathcal{O}(\hat{\phi}, \hat{\pi})$ is defined as

$$
\begin{aligned}
\langle\mathcal{O}(\hat{\phi}, \hat{\pi})\rangle_{\xi} \equiv & \int \prod_{i=1}^{N^{3}} d[\xi(t)]_{i} \mathcal{O}(\hat{\phi}, \hat{\pi}) \\
& \times \exp \left\{-\frac{a^{3}}{2 \Omega} \sum_{j=1}^{N^{3}} \int d t^{\prime} \xi_{j}^{2}\left(t^{\prime}\right)\right\}
\end{aligned}
$$

where the integration measure is normalized to give $\langle 1\rangle_{\xi}=1$. The probability density is a solution of the FP equation,

$\frac{\partial}{\partial t} P\left(\phi, \pi, t \mid \phi_{0}, \pi_{0}, t_{0}\right)=-\mathcal{L}_{\mathrm{FP}} P\left(\phi, \pi, t \mid \phi_{0}, \pi_{0}, t_{0}\right)$,

where the FP operator is defined as

$$
\begin{aligned}
\mathcal{L}_{\mathrm{FP}} \equiv & -a^{3} \sum_{i=1}^{N^{3}}\left\{-\pi_{i} \frac{\partial}{a^{3} \partial \phi_{i}}+\frac{\partial}{a^{3} \partial \pi_{i}}\left[\eta \pi_{i}-\nabla_{i j}^{2} \phi_{j}+V^{\prime}\left(\phi_{i}\right)\right]\right. \\
& \left.+\frac{\Omega}{2} \frac{\partial^{2}}{a^{6} \partial \pi_{i}^{2}}\right\} .
\end{aligned}
$$

\section{The probability density current}

Due to the conservation of probability, the FP equation can be written in terms of a probability density current $J$,

$\partial_{t} P(\phi, \pi, t)=-a^{3} \sum_{i=1}^{N^{3}} \frac{\partial}{a^{3} \partial \phi_{i}} J_{i}-a^{3} \sum_{i=1}^{N^{3}} \frac{\partial}{a^{3} \partial \pi_{i}} \bar{J}_{i}$,

where the components $J_{i}$ and $\bar{J}_{i}$ of the current are defined as

$$
J_{i} \equiv-\left\{-\pi_{i}-k_{B} T \frac{\partial}{a^{3} \partial \pi_{i}}\right\} P\left(\phi, \pi, t \mid \phi_{0}, \pi_{0}, t\right),
$$

and

$$
\begin{aligned}
\bar{J}_{i} \equiv & -\left\{\left[\eta \pi_{i}-\nabla_{i j}^{2} \phi_{j}+V^{\prime}\left(\phi_{i}\right)\right]+k_{B} T \frac{\partial}{a^{3} \partial \phi_{i}}\right. \\
& \left.+\frac{\Omega}{2} \frac{\partial}{a^{3} \partial \pi_{i}}\right\} P\left(\phi, \pi, t \mid \phi_{0}, \pi_{0}, t\right),
\end{aligned}
$$

for $i \in\left[1, N^{3}\right]$. The validity of this equation can be shown explicitly by substituting in Eq. (3.11).

\section{The equilibrium distribution}

The FP equation admits an equilibrium solution $P_{0}$ given by

$$
P_{0}(\phi, \pi)=\mathcal{Z}^{-1} \exp \{-\beta E[\phi, \pi]\},
$$

where $\mathcal{Z}$ is the normalization given by the partition function 


$$
\mathcal{Z}=\int \prod_{i=1}^{N^{3}} d \phi_{i} d \pi_{i} \exp \{-\beta E[\phi, \pi]\}
$$

and $E[\phi, \pi]$ is

$$
E[\phi, \pi]=a^{3} \sum_{i=1}^{N^{3}}\left[\frac{1}{2} \pi_{i}^{2}+\frac{1}{2}\left(\nabla \phi_{i}\right)^{2}+V\left(\phi_{i}\right)\right],
$$

which corresponds to the energy function of the system in the nondissipative limit.

\section{The vector-matrix notation}

Following the work of Langer [18], it is useful to introduce a vector-matrix notation. The field and its conjugate momentum are written in a $2 N^{3}$-dimensional vector as

$$
\left(\begin{array}{l}
\phi \\
\pi
\end{array}\right)=\left(\begin{array}{c}
\phi_{i}(t) \\
\pi_{i}(t)
\end{array}\right), \quad \text { where } i \in\left[1, N^{3}\right] .
$$

The deterministic limit of the Langevin equation is expressed as

$$
\frac{\partial}{\partial t}\left(\begin{array}{l}
\phi \\
\pi
\end{array}\right)=-M \cdot\left(\begin{array}{c}
\frac{\partial}{a^{3} \partial \phi} \\
\frac{\partial}{a^{3} \partial \pi}
\end{array}\right) E[\phi, \pi],
$$

with $M=\left(M_{i j}\right)$ being the $2 N^{3} \times 2 N^{3}$ block matrix defined as

$$
M=\frac{1}{a^{3}}\left(\begin{array}{cc}
0 & -\mathbb{1} \\
\mathbb{1} & \eta \mathbb{1}
\end{array}\right),
$$

where $\mathbb{1}$ is the $N^{3}$-dimensional unit matrix and the multiplication - between two $2 N^{3} \times 2 N^{3}$ matrices is defined as

$$
(A \cdot B)_{i j} \equiv a^{3} \sum_{k=1}^{2 N^{3}} A_{i k} B_{k j}
$$

A similar rule applies to the scalar product. The FP equation is given as

$$
\partial_{t} P(\phi, \pi, t)=-\left(\begin{array}{c}
\frac{1}{a^{3}} \frac{\partial}{\partial \phi} \\
\frac{1}{a^{3}} \frac{\partial}{\partial \pi}
\end{array}\right)^{T} \cdot\left(\begin{array}{l}
J \\
\bar{J}
\end{array}\right),
$$

where $(J \bar{J})^{T}$ is the $2 N^{3}$-dimensional vector corresponding to the probability current,

$$
\left(\begin{array}{l}
J \\
\bar{J}
\end{array}\right)=-M \cdot\left(\begin{array}{l}
\frac{1}{a^{3}} \frac{\partial E}{\partial \phi}+\frac{k_{B} T}{a^{3}} \frac{\partial}{\partial \phi} \\
\frac{1}{a^{3}} \frac{\partial E}{\partial \pi}+\frac{k_{B} T}{a^{3}} \frac{\partial}{\partial \pi}
\end{array}\right) P\left(\phi, \pi, t \mid \phi_{0}, \pi_{0}, t\right) .
$$

\section{The continuum limit}

We have been working in discrete space to simplify the analytical computations. However, the continuum limit can be taken at any stage of the derivation. For completeness, let us state the main quantities as expressed in the continuum limit. The FP equation reads

$\frac{\partial}{\partial t} P\left(\phi, \pi, t \mid \phi_{0}, \pi_{0}, t_{0}\right)=-\mathcal{L}_{\mathrm{FP}} P\left(\phi, \pi, t \mid \phi_{0}, \pi_{0}, t_{0}\right)$,

with

$$
\begin{aligned}
\mathcal{L}_{\mathrm{FP}} \equiv & -\int d^{3} \vec{x}\left\{-\pi(\vec{x}) \frac{\delta}{\delta \phi(\vec{x})}\right. \\
& \left.\left.+\frac{\delta}{\delta \pi(\vec{x})}\left[\eta \pi(\vec{x})-\nabla^{2} \phi(\vec{x})+V^{\prime} \phi\right)\right]+\frac{\Omega}{2} \frac{\delta^{2}}{\delta \pi(\vec{x})^{2}}\right\}
\end{aligned}
$$

and the equilibrium distribution is given by

$$
P_{0}(\phi, \pi)=\mathcal{Z}^{-1} \exp \{-\beta E[\phi, \pi]\},
$$

with

$$
\mathcal{Z}=\int D \phi D \pi \exp \{-\beta E[\phi, \pi]\}
$$

and

$E[\phi, \pi]=\int d^{3} \vec{x}\left[\frac{1}{2} \pi(\vec{x})^{2}+\frac{1}{2}(\nabla \phi(\vec{x}))^{2}+V(\phi)\right]$

is the energy functional.

\section{Computation of the rate}

The computation of the escape rate for the scalar field is a generalization of the zero-dimensional flux-overpopulation method to stochastic field theory. The original extension of the method to a $2 N$-dimensional system has been performed by Langer in Refs. [17,18].

\section{Setting up the problem}

The flux-over-population method relies on similar ideas as in the zero-dimensional case. The initial configuration is a homogeneous and static field located at the local minimum of the potential,

$$
\phi_{i}\left(t_{0}\right)=\phi_{i}^{A}, \quad \pi_{i}\left(t_{0}\right)=0, \quad \forall i .
$$

We assume that, for large negative values of the field, the potential is diverging and, on the other side, there is a local maximum located at $\phi^{B}$, as shown in Fig. 3. The probability density at time $t_{0}$ is a product of Dirac delta-functions peaked at $\phi=\phi^{A}$ and $\pi=0$, 


$$
P\left(\phi, \pi, t_{0} \mid \phi_{0}, \pi_{0}, t_{0}\right)=\prod_{i=1}^{N^{3}} \delta\left[\pi_{i}\right] \cdot \delta\left[\phi_{i}-\phi^{A}\right] .
$$

After a sufficiently long time, the system is expected to be described by the equilibrium distribution given in Eq. (3.14). At its early stages, the evolution of the system implies an increasing probability to find the field on the other side of the potential and, therefore, a flux of probability at the barrier.

The probability current is expected to go along the configuration with the minimal energy on the barrier ridge. This defines the saddle-point configuration, which is found by taking the variation of the energy function

$$
\delta E=a^{3} \sum_{i=1}^{N^{3}} \pi_{i} \delta \pi_{i}+\left[-\nabla^{2} \phi_{i}+V^{\prime}\left(\phi_{i}\right)\right] \delta \phi_{i} .
$$

We directly observe that the initial configuration is an extremum of the energy. The next configuration that extremizes the energy is given by $\pi_{i}^{S}=0$ and $\phi_{i}^{S}$ that satisfies the saddle-point equation,

$$
\nabla^{2} \phi_{i}^{S}=V^{\prime}\left(\phi_{i}^{S}\right)
$$

and defines the saddle-point configuration. The exact form of the solution $\phi^{S}$ is a priori not obvious. As stated in Sec. III A, a simple solution is the homogeneous case where the field is at the top of the potential $\phi^{B}$, at each point of space. This trivial solution of the saddle-point equation is relevant in a situation where the volume of space in consideration is finite. An example is the early Universe where this solution corresponds to the Hawking-Moss instanton [19], and the volume is a sphere of Hubble radius. In our case, the volume of space might be arbitrarily large. It is fair to assume that the rate of escape, which has an exponential dependence on the volume of space that experiences hopping, will be strongly suppressed when considering a large or even infinite volume. We, therefore, seek for a solution of the saddle-point equation where only a finite region of the space escapes, as already suggested in Ref. [16].

We might try to find a solution of Eq. (3.31) where the field is homogeneously sitting at the initial position $\phi^{A}$ everywhere except in some finite part, where it is climbing the potential well. Using the rotational symmetry and writing the saddle-point equation in spherical coordinates, we obtain

$$
\frac{\partial^{2}}{\partial r^{2}} \phi^{S}+\frac{2}{r} \frac{\partial}{\partial r} \phi^{S}=V^{\prime}\left(\phi^{S}\right),
$$

where, for simplicity, we are working in the continuum limit. The boundary conditions are

$$
\lim _{r \rightarrow \infty} \phi^{S}(r)=\phi^{A},\left.\quad \frac{\partial}{\partial r} \phi^{S}\right|_{r=0}=0,
$$

where the second condition has been introduced to make sure the left-hand side of the saddle-point equation (3.32) is finite at the center of the coordinates. The equation can be interpreted as the equation of motion of a fictitious point particle, in an inverted potential $-V$ and with a damping term. The overshoot/undershoot technique of Coleman [10] shows that a solution only exists if the original minimum is a false vacuum. We should then consider separately the cases where the initial minimum is a false (given by the dotted and dashed-dotted lines shown in Fig. 3) or a true (e.g., the dashed line shown in Fig. 3) vacuum.

In the case of a false vacuum at $\phi^{A}$, the saddle-point solution satisfying Eq. (3.32) exists and it is well understood. Let us consider the two limiting cases. If the potential is unbounded from below, by continuity, there must be a field value $\phi^{0}>\phi^{U 0}$, where the fictitious particle starts at $r=0$ with zero velocity and reaches $\phi^{A}$ at infinite radius. Moreover, it has been shown in Ref. [27] that $\phi^{0}$ is of the order of $\phi^{U 0}$. In the presence of a true vacuum at $\phi^{T V}$, the existence of a solution is ensured by the overshoot/ undershoot argument. If $V(\phi(r=0))>V\left(\phi^{A}\right)$, the fictitious particle does not have enough potential energy to climb the inverted potential up to $\phi^{A}$; this is an undershoot. On the other hand, if $\phi(r=0)$ is close enough to $\phi^{T V}$, the fictitious particle can stay near the true minimum until the damping term becomes negligible, since it is suppressed by $r$, and then it will overshoot. By continuity, there is a field value to start at $r=0$ that satisfies $V(\phi(r=0))<V\left(\phi^{A}\right)$ and $\phi(r=0)<\phi^{T V}$ such that the fictitious particle ends at $\phi^{A}$ at infinite radius. By these arguments, the saddle-point configuration is uniquely defined as well as the region of space that experiences hopping. Moreover, it has been shown by Coleman in Ref. [28] that the Hessian matrix of the energy evaluated for this configuration has only one negative eigenvalue. In the presence of an initial false vacuum, the escape rate is therefore analogous to the rate of nucleation of a critical bubble of true vacuum, due to thermal fluctuations, but now with in addition the damping explicitly taken into account.

One of the main differences with nucleation is that the escape problem can be defined for an initial true vacuum at $\phi^{A}$. However, a proper definition of the escape rate in this case requires additional care. On the one hand, by comparing the initial and the equilibrium distributions, it is fair to assume that there is a probability flow at the potential barrier and, therefore, it should be possible to define an escape. On the other hand, the undershoot argument forbids the existence of a solution of the saddle-point equation. We will come back to this issue at the end of this section, in Sec. III C 7, and make some propositions for a well-defined escape problem. For the moment, we simply assume that 
the initial position $\phi^{A}$ is a false vacuum and proceed with the computation of the escape rate. Note that the fact that the escape rate depends on the initial minimum being global or local is a clear difference with the point particle case discussed in Sec. II. For a field, the shape of the potential beyond the barrier plays a role in the evaluation of the escape rate.

The flux-over-population method relies on the following assumptions:

(i) No sources or sinks lie in the neighborhood of the saddle-point configuration. This allows us to write the FP equation (3.9) near the saddle point as

$$
\begin{aligned}
& a^{3} \sum_{i=1}^{N^{3}}\left\{-\pi_{i} \frac{\partial}{a^{3} \partial \phi_{i}}\right. \\
& \quad+\frac{\partial}{a^{3} \partial \pi_{i}}\left[\eta \pi_{i}+a^{3} \sum_{k=1}^{N^{3}}\left[-\frac{\nabla_{i k}^{2}}{a^{3}}+\frac{V^{\prime \prime}\left(\phi_{k}^{S}\right) \delta_{i k}}{a^{3}}\right]\right. \\
& \left.\left.\quad \times\left(\phi_{k}-\phi_{k}^{S}\right)\right]+\frac{\Omega}{2} \frac{\partial^{2}}{a^{6} \partial \pi_{i}^{2}}\right\} P(\phi, \pi)=0,
\end{aligned}
$$

using the expansion of the energy near the saddle point

$$
\begin{aligned}
E[\phi, \pi]= & E\left[\phi^{S}, \pi^{S}\right]+\frac{1}{2} a^{6} \sum_{i, j=1}^{N^{3}}\left(\phi_{i}-\phi_{i}^{S}\right) \\
& \times\left[-\frac{\nabla_{i j}^{2}}{a^{3}}+\frac{V^{\prime \prime}\left(\phi_{i}^{S}\right) \delta_{i j}}{a^{3}}\right]\left(\phi_{j}-\phi_{j}^{S}\right) \\
& +\frac{1}{2} a^{6} \sum_{i j=1}^{N^{3}}\left(\pi_{i}-\pi_{i}^{S}\right) \frac{\delta_{i j}}{a^{3}}\left(\pi_{j}-\pi_{j}^{S}\right)+\cdots
\end{aligned}
$$

In the spirit of the vector-matrix notation defined above, we introduce the matrix $\left(e_{i j}^{S}\right)$

$$
\left(e_{i j}^{S}\right)=-\frac{1}{a^{3}}\left(\begin{array}{cc}
-\nabla_{i j}^{2}+V^{\prime \prime}\left(\phi_{k}^{S}\right) \delta_{i j} & 0 \\
0 & \mathbb{1}
\end{array}\right),
$$

which corresponds to the negative of the Hessian matrix of the energy evaluated at the saddle-point configuration. In the context of field theory, the Hessian matrix is usually referred to as a fluctuation operator.

(ii) Inside the well, near the minimum where the field is located initially, the system is thermalized,

$$
P\left(\phi \simeq \phi^{A}, \pi \simeq \pi^{A}\right) \simeq P_{0}(\phi, \pi),
$$

where $P_{0}$ is the equilibrium distribution.

(iii) Beyond the saddle point, the probability density is strongly suppressed due to the presence of the sinks.

\section{The derivation of the probability density}

The computation of the flow of the probability current and the number density relies on the solution $P(\phi, \pi)$ of the FP equation with the boundary conditions given above. This solution is derived using the Kramers ansatz,

$$
P(\phi, \pi)=\zeta(\phi, \pi) P_{0}(\phi, \pi),
$$

where $\zeta(\phi, \pi)$ must be fixed to satisfy the boundary conditions,

$\zeta\left(\phi \simeq \phi^{A}, \pi \simeq \pi^{A}\right)=1, \quad \zeta\left(\phi>\phi^{S}, \pi\right) \rightarrow 0$.

The equation for $\zeta(\phi, \pi)$ is found by insertion in the FP equation. In particular, near the saddle point, one finds

$$
\begin{aligned}
& a^{3} \sum_{i=1}^{N^{3}}\left\{-\pi_{i} \frac{\partial}{a^{3} \partial \phi_{i}}\right. \\
& +\left[-\eta \pi_{i}+a^{3} \sum_{k=1}^{N^{3}}\left[-\frac{\nabla_{i k}^{2}}{a^{3}}+\frac{V^{\prime \prime}\left(\phi_{k}^{S}\right) \delta_{i k}}{a^{3}}\right]\left(\phi_{k}-\phi_{k}^{S}\right)\right] \frac{\partial}{a^{3} \partial \pi_{i}} \\
& \left.\quad+\frac{\Omega}{2} \frac{\partial^{2}}{a^{6} \partial \pi_{i}^{2}}\right\} \zeta(\phi, \pi)=0 .
\end{aligned}
$$

With the same arguments as in the zero-dimensional point particle case and following the Kramers original proposal, it is assumed that $\zeta(\phi, \pi)$ depends on a linear combination $u$ of the $\phi_{i}$ and $\pi_{i}$,

$$
\zeta(\phi, \pi)=\zeta(u),
$$

with

$$
u=a^{3} \sum_{i=1}^{N^{3}}\left[U_{i}\left(\phi_{i}-\phi_{i}^{S}\right)+\bar{U}_{i}\left(\pi_{i}-\pi_{i}^{S}\right)\right],
$$

where $U_{i}$ and $\bar{U}_{i}$ are the coefficients associated with $\phi_{i}$ and $\pi_{i}$, respectively. Similar arguments as the ones stated after Eq. (2.22) for the zero-dimensional point particle case justify this form of solution $\zeta(u)$. The following ansatz for $\zeta(u)$,

$$
\zeta(u)=\frac{1}{\sqrt{2 \pi k_{B} T}} \int_{u}^{\infty} d z \exp \left\{-\frac{z^{2}}{2 k_{B} T}\right\},
$$

satisfies the boundary conditions. To compute the coefficients $U_{i}$ and $\bar{U}_{i}$, we substitute $\zeta(u)$ in Eq. (3.40) and obtain 


$$
\begin{aligned}
& a^{3} \sum_{i=1}^{N^{3}}\left\{\left(U_{i}+\eta \bar{U}_{i}\right) \pi_{i}\right. \\
& -\bar{U}_{i} a^{3} \sum_{k=1}^{N^{3}}\left[-\frac{\nabla_{i k}^{2}}{a^{3}}+\frac{V^{\prime \prime}\left(\phi_{k}^{S}\right) \delta_{i k}}{a^{3}}\right]\left(\phi_{k}-\phi_{k}^{S}\right) \\
& \left.+\eta \bar{U}_{i}^{2} a^{3} \sum_{k=1}^{N^{3}} U_{k}\left(\phi_{k}-\phi_{k}^{S}\right)+\eta \bar{U}_{i}^{2} a^{3} \sum_{k=1}^{N^{3}} \bar{U}_{k}\left(\pi_{k}-\pi_{k}^{S}\right)\right\}=0 .
\end{aligned}
$$

At first sight, this equation seems unpromising. Fortunately, it can be written in a simple form using the vector-matrix notation that has been introduced previously. Defining the $\left(2 N^{3}\right)$ vectors $(U \bar{U})^{T}$ and $\left(\phi-\phi^{S} \pi-\pi^{S}\right)^{T}$ such that

$$
\begin{aligned}
u & =(U \bar{U}) \cdot\left(\begin{array}{c}
\phi-\phi^{S} \\
\pi-\pi^{S}
\end{array}\right) \\
& =a^{3} \sum_{i=1}^{N^{3}}\left[U_{i}\left(\phi_{i}-\phi_{i}^{S}\right)+\bar{U}_{i}\left(\pi_{i}-\pi_{i}^{S}\right)\right],
\end{aligned}
$$

with the scalar product being defined as in Eq. (3.20), the equation for the parameters $U_{i}$ and $\bar{U}_{i}$ becomes

$$
(U \bar{U}) \cdot M^{T} \cdot\left(e_{i j}^{S}\right) \cdot\left(\begin{array}{c}
\phi-\phi^{S} \\
\pi-\pi^{S}
\end{array}\right)=\lambda(U \bar{U}) \cdot\left(\begin{array}{c}
\phi-\phi^{S} \\
\pi-\pi^{S}
\end{array}\right),
$$

where the scalar $\lambda$ is defined as

$$
\lambda \equiv(U \bar{U}) \cdot M \cdot\left(\begin{array}{c}
U \\
\bar{U}
\end{array}\right)=a^{3} \sum_{i=1}^{N^{3}} \eta \bar{U}_{i} \bar{U}_{i} .
$$

The matrix equation (3.46) leads to the eigenvalue equation for $(U \bar{U})$,

$$
(U \bar{U}) \cdot M^{T} \cdot\left(e_{i j}^{S}\right)=\lambda(U \bar{U})
$$

and the other term $(U \bar{U})^{T}$ is a left eigenvector of the matrix $M^{T} \cdot\left(e_{i j}^{S}\right)$ with eigenvalue $\lambda$. Combining the definition of $\lambda$ and the eigenvalue equation, we find the normalization condition

$$
1=(U \bar{U}) \cdot\left(e_{i j}^{S}\right)^{-1} \cdot\left(\begin{array}{c}
U \\
\bar{U}
\end{array}\right)
$$

The eigenvalue $\lambda$ is positive by definition. The positivity is, in fact, a direct consequence of the overall negativity of the exponent of $\zeta(u)$. This negative exponent has been chosen in order to satisfy the boundary condition imposed by the method, namely the suppression of the probability distribution beyond the saddle point, and $\lambda$ is the only positive eigenvalue of the matrix $M^{T} \cdot\left(e_{i j}^{S}\right)$. Recall that $\left(e_{i j}^{S}\right)$ is defined as the negative of the Hessian of the energy, evaluated exactly at the saddle point.

\section{The probability density current and flux}

Once we have obtained the probability density $P=\zeta P_{0}$ we are ready to compute the associated probability density current defined in Eqs. (3.12) and (3.13). After some algebra, we find

$$
J \zeta P_{0}=\sqrt{\frac{k_{B} T}{2 \pi}} M \cdot\left(\begin{array}{c}
U \\
\bar{U}
\end{array}\right) \exp \left\{-\frac{u^{2}}{2 k_{B} T}\right\} P_{0},
$$

and the probability flux $j$ is given by

$$
\begin{aligned}
j= & a^{3} \sum_{i=1}^{2 N^{3}} \int_{u=0} d S_{i} J_{i}(\phi, \pi) \\
= & \frac{\lambda}{2 \pi \mathcal{Z}} \sqrt{\frac{k_{B} T}{2 \pi}} \exp \left\{-\beta E\left[\phi^{S}, \pi^{S}\right]\right\} \\
& \times \int D \phi D \pi \int d k \exp \left\{i k(U \bar{U}) \cdot\left(\begin{array}{c}
\phi-\phi^{S} \\
\pi-\pi^{S}
\end{array}\right)\right\} \\
& \times \exp \left\{\frac{\beta}{2}\left(\begin{array}{c}
\phi-\phi^{S} \\
\pi-\pi^{S}
\end{array}\right)^{T} \cdot\left(e_{i j}^{S}\right) \cdot\left(\begin{array}{c}
\phi-\phi^{S} \\
\pi-\pi^{S}
\end{array}\right)\right\} .
\end{aligned}
$$

We can diagonalize the matrix $\left(e_{i j}^{S}\right)$ by introducing the rotation $S=\left(S_{i j}\right)$ in field space to obtain

$$
\begin{array}{r}
\left(\begin{array}{c}
\phi-\phi^{S} \\
\pi-\pi^{S}
\end{array}\right)=S \cdot \xi, \\
i k u=i k(U \bar{U}) \cdot S^{\dagger} \cdot S \cdot\left(\begin{array}{c}
\phi-\phi^{S} \\
\pi-\pi^{S}
\end{array}\right)=i k \tilde{U} \cdot \xi,
\end{array}
$$

where we have defined the vector $\tilde{U}$ as $S \cdot(U \bar{U})^{T}$ and

$$
\left(\begin{array}{c}
\phi-\phi^{S} \\
\pi-\pi^{S}
\end{array}\right)^{T} \cdot\left(e_{i j}^{S}\right) \cdot\left(\begin{array}{c}
\phi-\phi^{S} \\
\pi-\pi^{S}
\end{array}\right)=a^{3} \mu_{1} \xi_{1}^{2}-a^{3} \sum_{l=2}^{2 N^{3}} \mu_{l} \xi_{l}^{2},
$$

where all the scalars $\mu_{l}$ are defined as positive. ${ }^{4}$ The only positive eigenvalue of $\left(e_{i j}^{S}\right)$ is $\mu_{1}$; all the other eigenvalues are $-\mu_{l}$. Hence, we finally can write the flux $j$ as

$$
j=\frac{\lambda}{2 \pi \mathcal{Z}} e^{-\beta E\left[\phi^{S}, \pi^{S}\right]}\left|\operatorname{det}(2 \pi / \beta)^{-1} E^{(S)}\right|^{-\frac{1}{2}},
$$

\footnotetext{
${ }^{4}$ For the moment, we ignore the possibility of vanishing eigenvalues. We shall come back to them shortly.
} 
where the matrix $E^{(S)}=-\left(e_{i j}^{S}\right)$ is the Hessian of the energy at the saddle point, and that has only one negative eigenvalue. Since this negative eigenvalue appears with a negative sign, it is the magnitude of the determinant that enters the formula. The successive integrations have been performed in the following order, first over all the modes $l$ larger than 1 , then over $k$ and finally over $\xi_{1}$.

\section{The zero modes}

Due to the translation invariance of the saddle-point solution, there are three eigenvalues in the associated determinant that are exactly zero and, therefore, must be treated separately upon the Gaussian integration. For simplicity and in order to agree with the literature, we perform the analysis in the continuum space. First of all, let us show that $\partial_{\vec{x}} \phi^{S}, \partial_{\vec{y}} \phi^{S}$ and $\partial_{\vec{z}} \phi^{S}$ are zero-modes. Considering $\partial_{\vec{x}} \phi^{S}$ we have

$$
\begin{aligned}
{\left[-\nabla^{2}+V^{\prime \prime}\left(\phi^{S}\right)\right] \partial_{\vec{x}} \phi^{S} } & =-\partial_{\vec{x}} \nabla^{2} \phi^{S}+V^{\prime \prime}\left(\phi^{S}\right) \partial_{\vec{x}} \phi^{S} \\
& =-\partial_{\vec{x}} V^{\prime}\left(\phi^{S}\right)+V^{\prime \prime}\left(\phi^{S}\right) \partial_{\vec{x}} \phi^{S} \\
& =-V^{\prime \prime}\left(\phi^{S}\right) \partial_{\vec{x}} \phi^{S}+V^{\prime \prime}\left(\phi^{S}\right) \partial_{\vec{x}} \phi^{S} \\
& =0 .
\end{aligned}
$$

To remove the zero-modes, we follow the procedure described in [18] and [11]. First of all, the determinant has its zero-eigenvalues removed and becomes

$$
\begin{aligned}
& \left|\operatorname{det}(2 \pi / \beta)^{-1}\left[-\nabla^{2}+V^{\prime \prime}\left(\phi_{i}^{S}\right)\right]\right| \\
& \quad \rightarrow\left|\operatorname{det}^{\prime}(2 \pi / \beta)^{-1}\left[-\nabla^{2}+V^{\prime \prime}\left(\phi_{i}^{S}\right)\right]\right|,
\end{aligned}
$$

with the prime denoting the removal of the vanishing eigenvalues. Then, the integration over the zero-modes $\partial_{\vec{x}} \phi^{S}, \partial_{\vec{y}} \phi^{S}$ and $\partial_{\vec{z}} \phi^{S}$ becomes an integration over $d \vec{x}, d \vec{y}$ and $d \vec{z}$, giving an overall volume factor $\mathcal{V}$. Finally each change of variable from the zero-modes to $\partial_{\vec{x}} \phi^{S}, \partial_{\vec{y}} \phi^{S}$ and $\partial_{\vec{z}} \phi^{S}$ to $d \vec{x}, d \vec{y}$ and $d \vec{z}$ leads to a Jacobian. For example, for the mode $\partial_{\vec{x}} \phi^{S}$ we have

$$
\left[\int d^{3} \vec{x}\left(\frac{\partial \phi^{S}}{\partial x}\right)^{2}\right]^{1 / 2}
$$

The Jacobian is identical for each zero-mode since

$$
\begin{aligned}
\int d^{3} \vec{x}\left(\frac{\partial \phi^{S}}{\partial x}\right)^{2} & =\int d^{3} \vec{x}\left(\frac{\partial \phi^{S}}{\partial y}\right)^{2} \\
& =\int d^{3} \vec{x}\left(\frac{\partial \phi^{S}}{\partial z}\right)^{2}
\end{aligned}
$$

where we used the rotation-symmetry of the saddle-point solution. We then have

$$
\int d^{3} \vec{x}\left(\frac{\partial \phi^{S}}{\partial x}\right)^{2}=\frac{1}{3} \int d^{3} \vec{x}\left(\nabla \phi^{S}\right)^{2}
$$

Hence, there is an overall factor multiplying the rate coming from the Jacobian and given by

$$
\left[\frac{1}{3} \int d^{3} \vec{x}\left(\nabla \phi^{S}\right)^{2}\right]^{3 / 2}
$$

A quick dimensional check tells us that removing the three eigenvalues from the determinant increases the dimension by $3 / 2$. The overall volume factor has a dimension of -3 and the Jacobian $3 / 2$, exactly compensating the removal of the zero-eigenvalues.

\section{Population inside the well}

The last missing piece is to account for the population inside the well. This is obtained using the condition that the system be thermalized near the minimum of the potential and by expanding the energy function around the configuration $\left(\phi^{A}, \pi^{A}\right)$,

$$
\begin{aligned}
E[\phi, \pi]= & E\left[\phi^{A}, \pi^{A}\right] \\
& +\frac{1}{2} a^{6} \sum_{i, j=1}^{N^{3}}\left(\phi_{i}-\phi_{i}^{A}\right)\left[-\frac{\nabla_{i j}^{2}}{a^{3}}+\frac{V^{\prime \prime}\left(\phi_{i}^{S}\right) \delta_{i j}}{a^{3}}\right]\left(\phi_{j}-\phi_{j}^{A}\right) \\
& +\frac{1}{2} a^{6} \sum_{i j=1}^{N^{3}}\left(\pi_{i}-\pi_{i}^{A}\right) \frac{\delta_{i j}}{a^{3}}\left(\pi_{j}-\pi_{j}^{A}\right)+\cdots
\end{aligned}
$$

The population $n_{A}$ inside the well is found to be given by

$$
\begin{aligned}
n_{A} & =\int D \phi D \pi P_{0} \\
& =\frac{1}{\mathcal{Z}} e^{-\beta E\left[\phi^{A}, \pi^{A}\right]}\left[\operatorname{det}(2 \pi / \beta)^{-1} E^{(A)}\right]^{-\frac{1}{2}},
\end{aligned}
$$

where the matrix $E^{(A)}$ is the Hessian of the energy of the initial configuration at $\phi^{A}$ and all eigenvalues are positive.

\section{The escape rate expression}

The ratio of the flux $j$ over the number density $n_{A}$, taking into account the zero-modes, gives the escape rate $k$ for a scalar field per unit volume,

$$
\begin{aligned}
\frac{k}{\mathcal{V}} & =\frac{\lambda}{2 \pi}\left[\frac{1}{3} a^{3} \sum_{i=1}^{N^{3}}\left(\nabla \phi_{i}^{S}\right)^{2}\right]^{3 / 2} \\
& \times\left[\frac{\operatorname{det}\left[(2 \pi / \beta)^{-1} E^{(A)}\right]}{\left|\operatorname{det}^{\prime}\left[(2 \pi / \beta)^{-1} E^{(S)}\right]\right|}\right]^{1 / 2} e^{-\beta\left[E\left(\phi^{S}, \pi^{S}\right)-E\left(\phi^{A}, \pi^{A}\right)\right]} .
\end{aligned}
$$

Let us consider the different contributions to the rate. In the exponent in Eq. (3.65) we have 


$$
\begin{aligned}
& E\left(\phi^{S}, \pi^{S}\right)-E\left(\phi^{A}, \pi^{A}\right) \\
& \quad=a^{3} \sum_{i=1}^{N^{3}} \frac{1}{2}\left(\nabla \phi_{i}^{S}\right)^{2}+V\left(\phi_{i}^{S}\right)-V\left(\phi_{i}^{A}\right),
\end{aligned}
$$

which corresponds to the activation energy, i.e., the difference between the energy of the saddle-point configuration with respect to the initial configuration. Since the initial configuration is homogeneous and only a difference of potential energy enters the rate formula, we can safely shift the potential to have $V\left(\phi_{i}^{A}\right)=0$. The determinants in Eq. (3.65) can be written as

$\operatorname{det}\left[(2 \pi / \beta)^{-1} E^{(A)}\right]=\operatorname{det}\left[(2 \pi / \beta)^{-1}\left(-\nabla^{2}+V_{A}^{\prime \prime}\right)\right]$,

where $V_{A}^{\prime \prime}$ is the second derivative of the potential at the initial minimum and

$$
\begin{aligned}
& \left|\operatorname{det}^{\prime}\left[(2 \pi / \beta)^{-1} E^{(S)}\right]\right| \\
& \quad=\left|\operatorname{det}^{\prime}(2 \pi / \beta)^{-1}\left[-\nabla^{2}+V^{\prime \prime}\left(\phi^{S}\right)\right]\right|,
\end{aligned}
$$

where the field configuration entering the operator is the saddle-point solution.

Substituting the above equations into Eq. (3.65), we then find that the escape rate per unit volume in the continuum limit is

$$
\begin{aligned}
\frac{k}{\mathcal{V}} & =\frac{\lambda}{2 \pi}\left[\frac{\beta}{6 \pi} \int d^{3} \vec{x}\left(\nabla \phi^{S}\right)^{2}\right]^{\frac{3}{2}} \\
& \times\left[\frac{\operatorname{det}\left[-\nabla^{2}+V_{A}^{\prime \prime}\right]}{\left|\operatorname{det}^{\prime}\left[-\nabla^{2}+V^{\prime \prime}\left(\phi^{S}\right)\right]\right|}\right]^{\frac{1}{2}} e^{-\beta \int d^{3} \vec{x}\left[\frac{1}{2}\left(\nabla \phi^{S}\right)^{2}+V\left(\phi^{S}\right)\right]},
\end{aligned}
$$

which, in conjunction with our derivation, is the main result of this paper. The constant $\lambda$ appearing in the above result is sometimes referred to as the dynamical prefactor and the ratio of determinants as the statistical prefactor $[8,13,18,29]$. The explicit expressions of these factors depend on the saddle-point configuration $\phi^{S}$. It should be noted that Eq. (3.69) has many similarities to the corresponding expression for the particle case Eq. (2.30). This should not be too surprising since escape rates are usually in the form of a statistical and a dynamical prefactor multiplying an exponential of the free energy associated with the escape. The difficulty lies in specifying the different prefactors and the free energy of the escape configuration. In particular, the dynamical prefactor $\lambda$ in Eq. (3.69) does not take such a simple form as in Eq. (2.25). The ratio of determinants and the Jacobian associated with the zero modes, which both form the statistical prefactor, are common in field theories. Finally, it of course must be kept in mind that the expression in the exponential arises from an integral over the whole field configuration. Methods specific to such a system and not just a point particle need to be utilized when evaluating, as is evidenced by the saddle-point configuration used above, which would not be possible for the point particle case.

We choose to present here the most general form of the escape rate. An explicit estimation of the rate, in particular the prefactors and the exponent, is possible once a potential has been specified. A discussion about the methods to estimate the rate in a practical case, for example the thinwall approximation, is given in Sec. IV B.

\section{The initial stable minimum}

The last case left to consider is when $\phi^{A}$ in Fig. 3 is a true vacuum. As described above, the saddle-point equation (3.32) does not have any solution. However, in the presence of fluctuation and dissipation dynamics, it is fair to assume, in any given realization of the noise, that the field starts to climb the potential and probes the other side of the well, even if it will likely come back to the original side. Moreover, as noted already, the comparison between the initial probability distribution, which is a Dirac delta function peaked at $\phi^{A}$ at each point in space, and the equilibrium distribution, that probes both sides of the well, implies a flow of probability through the maximum of the potential. These two arguments suggest that the escape problem for an initial true vacuum might still be defined. The rate will simply indicate how likely it is to have a region of space that passes the barrier. Let us formulate some propositions for a meaningful definition for this case.

The first possibility for treating the present case is to consider a finite volume $\mathcal{V}$ of space and use the saddle-point solution $\phi^{S}=\phi^{B}$ at each point in the volume. The activation energy will be given by $E=\mathcal{V} \Delta V$. This is the simplest generalization of the zero-dimensional case but it is dependent on the volume in consideration. Moreover, it can lead to an underestimate of the rate since, instead of waiting at the top of the potential, the field can fall on the other side and attract the neighboring points without any additional energy.

The method of reactive flux, described, for example, in the review [8], might be helpful in the derivation of the escape rate for an initially true minimum. At equilibrium, the ratio of particle densities in the wells is equal to the ratio of the rates between the two minima. Since the equilibrium distribution and the rate from a false to a true vacuum are known, the transition rate from an initial true vacuum can be extracted. It is reasonable to assume that, at equilibrium, the activation rate derived with the method of reactive flux will be smaller than the true escape rate. However, this method also allows us to study further the approach to equilibrium by defining a relaxation rate, from an initial out-of-equilibrium distribution.

Alternatively, we can consider an approximated case, where, in Fig. 3, the false minimum on the right-hand side is replaced by a true minimum, due to a modification of the potential beyond the maximum. For example, a minimal situation could be a new true minimum, almost degenerate 
with $V\left(\phi^{A}\right)$. A saddle-point configuration is well defined and the rate is given by Eq. (3.69). Moreover, the saddlepoint configuration will naturally define the typical size of the region of space that experiences hopping. As in the previous case, the escape rate might be underestimated. However, it is fair to expect that the main contribution to the escape time is given by the climbing of the potential well, which corresponds to the part of the potential that is not modified.

The last possibility is the construction of a saddle-point configuration using an analytic continuation. It was not possible to obtain a solution of Eq. (3.32), where the field is at $\phi^{F V}$ at $r=0$ and respecting the boundary condition (3.33). One can imagine giving an initial imaginary velocity to the field, which would then allow for the climb. This kind of solution has been studied in the context of tunneling [30-34]. However, this goes beyond the scope of this work, and we leave it for a future analysis.

\section{DISCUSSION OF THE RESULT}

Let us present here a comparison between the result we have obtained for the escape rate, given by Eq. (3.69), and the related problem of quantum tunneling at a sufficiently high temperature, where thermal effects dominate. The similarities between the two results provide some insights about the methods needed for an explicit evaluation of the escape rate, once a potential has been specified.

\section{A. Comparison with quantum tunneling at finite temperature}

Quantum tunneling of a scalar field is a well-studied problem and plays a significant role in the study of firstorder phase transitions and in the stability of false vacua. The problem has been solved for quantum field theory by Callan and Coleman at zero temperature $[10,11]$ and later extended to finite temperatures by Linde [12]. The result of Ref. [12] is particularly interesting for the current analysis since, for sufficiently high temperatures where the thermal fluctuations dominate over the quantum fluctuations, it recovers the result of Langer for classical nucleation $[17,18]$. In this regime, it is fair to expect some similarities between the tunneling and the escape rates.

The quantum tunneling rate per unit volume, at finite temperature and when thermal fluctuations are dominant, is given by

$$
\begin{aligned}
\frac{\Gamma(T)}{\mathcal{V}}= & T\left(\frac{\mathcal{S}_{3}\left(\phi^{S}, T\right)}{2 \pi T}\right)^{\frac{3}{2}}\left[\frac{\operatorname{det}\left[-\nabla^{2}+V_{A}^{\prime \prime}\right]}{\left|\operatorname{det}^{\prime}\left[-\nabla^{2}+V^{\prime \prime}\left(\phi^{S}\right)\right]\right|}\right]^{\frac{1}{2}} \\
& \times \exp \left\{-\mathcal{S}_{3}\left(\phi^{S}, T\right) / T\right\},
\end{aligned}
$$

where the action $\mathcal{S}_{3}$ is defined as

$$
\mathcal{S}_{3}(\phi, T) \equiv \int d^{3} \vec{x}\left[\frac{1}{2}(\nabla \phi)^{2}+V(\phi, T)\right],
$$

and $\phi^{S}$ is a solution of

$$
\frac{\partial^{2}}{\partial r^{2}} \phi^{S}+\frac{2}{r} \frac{\partial}{\partial r} \phi^{S}=V^{\prime}\left(\phi^{S}, T\right),
$$

where $V(\phi, T)$ is the temperature-dependent effective potential.

Comparing with the escape problem, and assuming identical potentials, ${ }^{5}$ we immediately notice that the field configurations entering the two rates are the same, Eq. (3.32) for the case of the escape problem and Eq. (4.3) given above. This similarity implies that the ratio of determinants and the exponential term are identical in the escape rates [Eq. (3.69)] and in the tunneling rates [Eq. (4.1)], respectively. Using the argument of Coleman $[10,35]$, that the action $\mathcal{S}_{3}$ is invariant under an infinitesimal scale transformation of the solution $\phi^{S}$, we obtain

$$
\mathcal{S}_{3}(\phi, T)=\frac{1}{3} \int d^{3} \vec{x}(\nabla \phi)^{2},
$$

which is precisely the term given by the Jacobian in the escape rate problem.

The crucial difference between the escape and the quantum tunneling rates lies in the prefactors. In particular, the escape rate predicts a factor of $\lambda / 2 \pi$ replacing the temperature. We interpret this difference as follows. First of all, the escape problem, even if closely related, is not defined exactly as the transition rate due to tunneling effects. A comparable, but not identical, rate should emerge. Moreover, to derive the escape rate, we used the framework of stochastic field theory, where the strength of the noise and the damping appear explicitly. One naturally expects the damping to play a role in the final result, in particular within the dynamical prefactor $\lambda$ in Eq. (3.69). On the contrary, the temperature that appears in the prefactor of (4.1) is an approximation which relies on dimensional grounds. In the approach of Ref. [12], the properties characterizing the medium, for example the viscosities, are not taken into account. When the properties of the medium are considered [13,36,37], a dynamical prefactor is expected in the rate.

It is however remarkable that the two rates computed with different methods, the stochastic field theory for the escape problem and the path integral formalism of quantum field theory for tunneling, have so much in common. The escape rate only takes into account the thermal fluctuations and is valid for arbitrarily small temperatures. It is a strong support for Eq. (3.69) that the tunneling rate, in the limit where the thermal fluctuations dominate, mostly recovers the escape rate.

\footnotetext{
${ }^{5}$ To be more precise, we assume that the potential of the escape rate $V(\phi)$ is equal to the effective potential $V(\phi, T)$ at a fixed value of $T$.
} 


\section{B. Towards an explicit evaluation of the escape rate}

In general, once a potential has been specified, a complete derivation of the escape rate, Eq. (3.69), requires numerical methods, as for example in Ref. [38]. However, exploiting the similarities with the quantum tunneling rate, we can use the techniques developed for the latter to provide some guidance on the explicit derivation of the escape rate. This holds even if the derivations of both rates, as stated before, are based on completely different methods, path integrals for tunneling and a stochastic approach for the escape rate. Let us consider the exponent, the dynamical and statistical prefactor terms appearing in our final result Eq. (3.69), separately. Recall that, in general, it is sufficient to know the order of magnitude of the prefactors, the rate being mainly dictated by the exponential.

\section{The exponent term}

The evaluation of the exponent term in Eq. (3.69) requires the solution of the saddle-point equation (3.32), which, in general, is obtained numerically. However, two cases have been identified where an analytical treatment is possible [12,27]. In the thin-wall approximation, the potential has two minima that are almost degenerate. The saddle-point configuration has the form of a bubble of true vacuum. Going along the radial direction, $\phi^{S}(r)$ is initially almost constant and close to $\phi^{T V}$. This corresponds to the interior of the bubble. The field solution then bounces to $\phi^{A}$, which defines the wall of the bubble. The critical radius of the bubble is found by minimizing the energy. It has been shown in $[12,27]$ that the exponent becomes

$$
\int d^{3} \vec{x}\left[\frac{1}{2}\left(\nabla \phi^{S}\right)^{2}+V\left(\phi^{S}\right)\right]=\frac{16 \pi}{3 \epsilon^{2}}\left(\int_{\phi^{T V}}^{\phi^{A}} d \phi \sqrt{2 V(\phi)}\right)^{3},
$$

where $\epsilon$ is the difference between the false and true vacua, and the integral on the right-hand side is evaluated in the limit where $\epsilon$ vanishes. The other situation where an analytical treatment is possible is when the potential difference between the false and true vacua is much larger than the barrier height. The potential can be approximated by a cubic or a quartic polynomial function in the field, leading to exact solutions.

\section{The statistical prefactor}

The exact evaluation of ratios of determinants in field theory is, in general, an involved task. Recent discussions on some analytical approaches to this problem can be found in [39-41]. For the evaluation of the escape rate just as for the tunneling case, as stated in [12,27], it is sufficient to have only a rough estimate of this prefactor. Dimensional analysis shows that the square root of the ratio of determinants has dimension $\mathrm{m}^{3}$ corresponding to the removal of the three eigenvalues in the denominator. Therefore, we can write

$$
\left[\frac{\operatorname{det}\left[-\nabla^{2}+V_{A}^{\prime \prime}\right]}{\left|\operatorname{det}^{\prime}\left[-\nabla^{2}+V^{\prime \prime}\left(\phi^{S}\right)\right]\right|}\right]^{\frac{1}{2}} \sim \mathcal{O}\left(\phi^{3},\left(V^{\prime \prime}\right)^{3 / 2}, r^{-3}, T^{3}\right),
$$

where the quantities on the right-hand side (apart from the temperature) should be understood as mean values. In general, $\phi^{3},\left(V^{\prime \prime}\right)^{3 / 2}$, and $r^{-3}$ are of the same order of magnitude and should be compared with the temperature to find the dominant contribution. This is different from the case of quantum tunneling at a finite temperature, where the temperature is expected to dominate in the statistical prefactor.

\section{The dynamical prefactor}

The dynamical prefactor $\lambda$ has been defined in Eq. (3.48) as the unique positive eigenvalue of the matrix $M^{T} \cdot\left(e_{i j}^{S}\right)$. The eigenvalue equation for $\lambda$ can be written as

$$
\left[\frac{\partial^{2}}{\partial r^{2}}+\frac{2}{r} \frac{\partial}{\partial r}-V^{\prime \prime}\left(\phi^{S}\right)\right] v(r)=\lambda(\lambda+\eta) v(r) .
$$

We observe that $\lambda$ has a dependence on the dissipation coefficient $\eta$. As usual, an analytical solution of the eigenvalue equation is not possible, in particular, since it requires the knowledge of the saddle-point configuration $\phi^{S}(r)$. There exists, however, certain situations where an approximate result might be obtained, for example in the thin-wall approximation discussed above. Useful discussions on this problem can be found in Refs. [13,29,36,37].

\section{POSSIBLE APPLICATIONS}

Here we will identify some suggested applications for the derived result for the escape problem in field theory. In fact, there can be applications in any situation involving a phase transition. In high-energy physics this could be in the context of cosmology as well as in heavy-ion collision experiments, and at low energy in condensed matter systems. In all these cases one can find applications where the escape problem defined here plays a relevant role. A particular interest is to look at scenarios where the escape rate provides an alternative mechanism to quantum tunneling. We also identify problems where the methods, developed here in order to derive the escape rate, provide an alternative approach. Since the aim of the current analysis is a formal definition and a solution of the Kramers problem, we restrict to a general description of these applications. A deeper analysis is left for future works.

a. Phase transitions and topological defects.-A concrete situation where the escape rate becomes significant is in the study of out-of-equilibrium systems, in particular, during a first-order phase transition. Our analysis is well 
suited to investigate the approach to equilibrium. We can imagine, for example, the situation of an initially quadratic effective potential that is developing another local minimum. The second minimum is, at first, a false vacuum before becoming the true vacuum of the potential. The escape rate provides the necessary tools to study the evolution of the FP probability distribution between the old and the new equilibrium distributions.

Phase transitions are often associated with the formation of topological defects $[42,43]$. Fluctuation and dissipation dynamics can influence their creation, in particular in a second-order phase transition, where the height of the potential barrier is suppressed at the beginning of the transition. These effects might also play an important role in crossover transitions. In the special case of embedded defects [44-47], the possibility for the field to escape would have some consequences on the stability of the configuration. Examples of realistic stable embedded defects are known [48]. The escape rate should, therefore, be related to the destruction probability of such a stable embedded configuration.

b. Landscape of metastable minima.-One of the most interesting features of the escape problem is the hopping of the field over the potential barrier. Naively, considering a potential with two minima that are almost degenerate, the escape rate between the false and the true vacua should not be sensibly different from the rate between the true and the false vacua. For these reasons, the escape rate could be relevant in theories that contain several nondegenerate minima, in particular, in order to compute the probability for a finite part of space to evolve from one minimum to the next. One can imagine, for example, a situation with two possible directions to diffuse. In one of them, there is a large potential barrier but a minimum at a lower energy beyond the well. In the other direction, the potential barrier is smaller but the next minimum is at a higher energy. Quantum tunneling could only be applied to the first case but the escape mechanism is applicable in both cases.

Such a situation arises in string theories, which contain many metastable vacua [49]. This framework is called the string landscape [50]. The question of how a vacuum is selected is of particular interest. Our mechanism precisely allows for the hopping from one vacuum to the next one. Moreover, the Hagedorn temperature [51,52], usually associated with string theories, could be the origin of the fluctuation and dissipation dynamics. Such an analysis might require a generalization of our work to take into account gravitational effects.

An active field of research in condensed matter physics concerns the glass transition [53], corresponding to a phase transition between a liquid and a glassy state. The phenomenology of glassy systems can be described by an $N$-body system in a potential with several metastable minima, called the potential energy landscape $[54,55]$. The escape rate provides a mechanism to probe the different minima. A generalization of our analysis to a nonrelativistic field would be needed in this case.

c. Stochastic inflation.-The stochastic formulation of inflation was introduced by Starobinsky [56,57] as a framework to study the dynamics of a quantum scalar field during inflation. The field is split into two parts, the longwavelength part (coarse grained) and short-wavelength quantum fluctuations. The backreaction of the quantum fluctuations on the coarse grained part is parametrized as a stochastic noise. The equation of motion of the inflaton becomes a Langevin equation. The framework is particularly relevant in the computation of correlation functions of the inflaton field [58].

In general, the noise is assumed to be homogeneous and the problem reduces to the zero-dimensional case described in Sec. II. This approach considers only the fluctuations that can lift an entire Hubble sphere. If, on the other hand, we imagine that the backreaction coming from the quantum fluctuations is inhomogeneous, the formalism developed for the escape rate is particularly useful. One can also think about different regions of space that evolve along different directions in the inflationary potential.

d. Stochastic quantization.-The stochastic approach of quantum mechanics was first proposed by Nelson in [59] and then extended to fields by Parisi and $\mathrm{Wu}$ in [60]. The main idea relies on the fact that the generating functional of Euclidean field theories is related to the equilibrium limit of a statistical system coupled to a heat reservoir. The temperature of the heat bath is chosen to match the Planck constant. The evolution of the system plus reservoir is in a fictitious time and the equilibrium is reached when this extra time direction goes to infinity. This method for modeling quantum field theory is particularly useful for numerical simulations, such as in lattice field theory [61].

The stochastic field theory introduced for the derivation of the escape rate is formally equivalent to the formalism describing stochastic quantization. The only difference is the dimension of space. The formalism described in Sec. III B can be seen as a three-dimensional Euclidean field theory coupled to a heat bath, whereas the stochastic quantization considers a four-dimensional Euclidean field theory and an extra time dimension. In the language of stochastic quantization, in particular, using the identification $\hbar=k_{B} T$, we can directly write the escape rate as

$\frac{k}{\mathcal{V}}=\frac{\lambda}{2 \pi}\left[\frac{\mathcal{S}_{4}}{2 \pi \hbar}\right]^{2}\left[\frac{\operatorname{det}\left[-\square+V_{A}^{\prime \prime}\right]}{\left|\operatorname{det}^{\prime}\left[-\square+V^{\prime \prime}\left(\phi^{S}\right)\right]\right|}\right]^{\frac{1}{2}} e^{-\mathcal{S}_{4}\left(\phi^{S}\right) / \hbar}$,

where

$$
\mathcal{S}_{4}(\phi) \equiv \int d^{4} \vec{x}\left[\frac{1}{2}(\nabla \phi)^{2}+V(\phi)\right]
$$

and $\phi^{S}$ is the saddle-point configuration. If the system is initially in a false vacuum, the quantum escape rate (5.1) 
defined for quantum fluctuations gives a quantum nucleation rate, which should be equivalent to quantum tunneling in the usual quantization. A similar treatment as in Sec. IV A should be performed to compare this result with the quantum tunneling rate at zero-temperature, computed in Refs. $[10,11]$, and to study how the two results agree. We leave the analysis of the quantum escape rate open for future works.

\section{CONCLUSION}

In this work, we have proposed a definition and a solution of the Kramers problem for a scalar field theory. Using the framework of stochastic field theory, we have studied the probability for a scalar field to escape a potential well due to thermal fluctuations. The field theory character of the problem complicates the definition of the escape configuration. Unlike the zero-dimensional point particle case, we have learned that the shape of the potential, beyond the local maximum, influences the rate. Two situations have been identified that need to be treated separately, when the initial minimum corresponds to a true or a false vacuum. Using a generalization of the flux-overpopulation method to a field, we have derived a full solution of the escape problem from a metastable vacuum and stated some directions to address the case of an initial true vacuum.

The main result of our analysis is the expression of the escape rate, Eq. (3.69). A comparison with the quantum tunneling rate, in the limit where the thermal fluctuations dominate, shows that the two rates have much in common. These similarities provide strong support for our result, in particular, since both rates are computed from different approaches. The rates are, however, not identical. This is not surprising, since the two problems, even if related, are not exactly the same. In particular, the escape rate explicitly takes damping effects into account. Nevertheless, the wellstudied framework of quantum tunneling provides some useful techniques for an explicit evaluation of the escape rate, once a potential is fixed. In this work, we have taken specific advantage of that. It is remarkable that the derivation presented in this paper also encompasses the Hawking-Moss instanton. This solution naturally emerges from the flux-over-population method and can be studied within the framework presented here.

Beyond the formal interest of the Kramers problem in field theory, we have identified several concrete situations, in cosmology, particle physics and condensed matter physics, where the escape rate is relevant. Out-of-equilibrium scenarios, for example during a transition between two nondegenerate vacua, are natural candidates. In cosmology, phase transitions and the formation of topological defects, as well as stochastic inflation, are various applications. The string landscape and the glass transition present a favorable environment for an escape mechanism. On a more formal level, the analogy with the stochastic quantization might shed new light on both the interpretation of the escape problem and on the meaning of the stochastic approach of quantum mechanics. A deeper analysis of these directions will require further work.

\section{ACKNOWLEDGMENTS}

A. B. is supported by STFC. J. M. is supported by Principal's Career Development Scholarship and Edinburgh Global Research Scholarship. B. W. M. and R. O.R. thank the Brazilian funding agencies CAPES, $\mathrm{CNPq}$ and FAPERJ for financial support. B. W. M. and R. O.R. are partially supported by research grants from Conselho Nacional de Desenvolvimento Científico e Tecnológico (CNPq), under Grants No. 431.796/2016-5 (B. W. M.) and No. 302545/2017-4 (R. O. R.) and also by Fundação Carlos Chagas Filho de Amparo à Pesquisa do Estado do Rio de Janeiro (FAPERJ), under Grants No. E-26/202.649/2018 (B. W. M.) and No. E-26/202.892/ 2017 (R. O. R.).

\section{APPENDIX: MEAN FIRST PASSAGE TIME}

\section{Definition of the MFPT over the barrier}

An alternative derivation of the escape rate is achieved with the method of the mean first passage time. The first passage time (FPT) is defined as the time the particle takes to leave a domain $\mathcal{D}$ for the first time. In our case, it corresponds to the time needed for the particle initially at $x_{A}$ to pass over the maximum at $x_{B}$ as depicted in Fig. 1. Since the forces acting on the particle are random and the dynamics not deterministic, the FPT is different for each realization. One can, however, define the MFPT as the average of the FPT and estimate the escape rate as its inverse.

A formal definition of the problem relies on the introduction of the survival probability $S\left(t \mid x_{0}, v_{0}, t_{0}\right)$. It corresponds to the probability that the particle is still in $\mathcal{D}$ after a time $\left(t-t_{0}\right)$, while being initially at position $x_{0}$ with velocity $v_{0}$. In our case, the domain is the $A$-well where $x \in\left(-\infty, x_{S i}\right]$, where the upper limit of the domain, $x_{S i}$, is a point chosen to be near, but beyond, the maximum, to ensure the passing of the particle. The survival probability is defined as

$$
\begin{aligned}
S\left(t \mid x_{0}, v_{0}, t_{0}\right) & =\int_{\mathcal{D}} d x d v P\left(x, v, t \mid x_{0}, v_{0}, t_{0}\right) \\
& =\operatorname{Prob}\left[T\left(x_{0}, v_{0}\right)>\left(t-t_{0}\right)\right] \\
& =\int_{\left(t-t_{0}\right)}^{\infty} d t f\left(t \mid x_{0}, v_{0}\right),
\end{aligned}
$$

where $T\left(x_{0}, v_{0}\right)$ is the FPT starting at $x_{0}$ with initial velocity $v_{0}$ and $f\left(t \mid x_{0}, v_{0}\right)$ is the probability distribution for $T\left(x_{0}, v_{0}\right)$. The above relation is motivated by the following reasoning. The probability to be in the domain 
at time $t$ is the same as the probability of having a first passage time larger than $\left(t-t_{0}\right)$.

From Eq. (A1), we deduce the following relation between $S\left(t \mid x_{0}, v_{0}, t_{0}\right)$ and $f\left(t \mid x_{0}, v_{0}\right)$ :

$$
f\left(t \mid x_{0}, v_{0}\right)=-\frac{\partial S\left(t \mid x_{0}, v_{0}, t_{0}\right)}{\partial t} .
$$

The moments $\left\langle T^{n}\right\rangle$ of the FPT are defined as

$$
\begin{aligned}
\left\langle T^{n}\right\rangle & \equiv \int_{t_{0}}^{\infty} d t\left(t-t_{0}\right)^{n} f\left(t \mid x_{0}, v_{0}\right) \\
& =n \int_{t_{0}}^{\infty} d t\left(t-t_{0}\right)^{n-1} S\left(t \mid x_{0}, v_{0}, t_{0}\right),
\end{aligned}
$$

and, in particular, the MFPT $\tau$ reads

$$
\begin{aligned}
\tau & \equiv\langle T\rangle=\int_{t_{0}}^{\infty} d t S\left(t \mid x_{0}, v_{0}, t_{0}\right) \\
& =\int_{t_{0}}^{\infty} d t \int_{\mathcal{D}} d x d v P\left(x, v, t \mid x_{0}, v_{0}, t_{0}\right) .
\end{aligned}
$$

We understand this expression for $\tau$ in the following way. The averaged first passage time is the sum of all the probabilities to be in the domain $\mathcal{D}$ at any time $t$ larger than $t_{0}$. If the particle is never in $\mathcal{D}$, the integrand vanishes and so does the MFPT. If, on the other hand, the particle is always in the domain, the integral over the probability distribution is normalized to 1 and the time integral diverges, leading to an infinite MFPT.

Using the adjoint FP equation, it is possible to find an explicit solution for the MFPT,

$$
\begin{aligned}
\mathcal{L}_{\mathrm{FP}}^{\dagger} \tau & =\int_{t_{0}}^{\infty} \int_{\mathcal{D}} d x d v \mathcal{L}_{\mathrm{FP}}^{\dagger} P\left(x, v, t \mid x_{0}, v_{0}, t_{0}\right) \\
& =-\left.\int_{\mathcal{D}} d x d v P\left(x, v, t \mid x_{0}, v_{0}, t_{0}\right)\right|_{t=t_{0}} ^{\infty}=1,
\end{aligned}
$$

where we assumed that the probability to be in the domain for $t$ going to infinity vanishes, and we used $P\left(x, v, t_{0} \mid x_{0}, v_{0}, t_{0}\right)=\delta\left(x-x_{0}\right) \delta\left(v-v_{0}\right)$. To find the mean first passage time, it is sufficient to solve $\mathcal{L}_{\mathrm{FP}}^{\dagger} \tau=1$ with the boundary condition $\tau=0$ on $\partial \mathcal{D}$. Despite the apparent simplicity of the equation describing the MFPT, the computation turns out to be rather involved in practice.

\section{Formal equivalence between the MFPT and the flux-over-population method}

A formal relationship between the flux-over-population and the MFPT methods has been shown in Refs. [8,9]. We have learned in the previous section that the MFPT $\tau_{\mathcal{D}}\left(x_{0}, v_{0}\right)$ is defined by the equation

$$
\mathcal{L}_{\mathrm{FP}}^{\dagger} \tau_{\mathcal{D}}\left(x_{0}, v_{0}\right)=1, \quad\left(x_{0}, v_{0}\right) \in \mathcal{D},
$$

and the boundary condition $\tau_{\mathcal{D}}\left(x_{0}, v_{0}\right)=0$ for $x_{0} \in \partial \mathcal{D}$. The Green's function $g\left(x, v_{x} \mid y, v_{y}\right)$ for the FP operator on $\mathcal{D}$ is defined as

$$
\mathcal{L}_{\mathrm{FP}}\left(x, v_{x}\right) g\left(x, v_{x} \mid y, v_{y}\right)=k \delta(x-y) \delta\left(v_{x}-v_{y}\right),
$$

for $\left(x, v_{x}\right) \in \mathcal{D}$, and

$$
g\left(x, v_{x} \mid y, v_{y}\right)=0, \quad x \in \partial \mathcal{D} .
$$

The Green's function might be interpreted as a stationary probability distribution, since it is a time-independent solution of the FP equation at every point of the phase space but $\left(y, v_{y}\right)$. This point might be seen as an additional point source of strength $k$. Moreover, the boundary $\mathcal{D}$ acts as a sink. The conservation of probability implies that the source strength is related to the probability to be absorbed per unit time, i.e.,

$$
\begin{aligned}
k & =\int_{\mathcal{D}} d x d v \mathcal{L}_{\mathrm{FP}}\left(x, v_{x}\right) g\left(x, v_{x} \mid y, v_{y}\right) \\
& =\int_{\partial \mathcal{D}} d S_{i} J_{i}\left(x, v_{x} \mid y, v_{y}\right),
\end{aligned}
$$

where $J_{i}$ is the probability current density defined from the FP equation,

$$
\mathcal{L}_{\mathrm{FP}}\left(x, v_{x}\right) g\left(x, v_{x} \mid y, v_{y}\right)=\frac{\partial}{\partial x} J_{x}+\frac{\partial}{\partial v_{x}} J_{v} .
$$

After a multiplication of the Green's function with the MFPT and the integration over the domain $\mathcal{D}$, we obtain

$$
\begin{aligned}
& \int_{\mathcal{D}} d x d v \tau_{\mathcal{D}\left(x, v_{x}\right)} \mathcal{L}_{\mathrm{FP}}\left(x, v_{x}\right) g\left(x, v_{x} \mid y, v_{y}\right) \\
& =k \int_{\mathcal{D}} d x d v \tau_{\mathcal{D}\left(x, v_{x}\right)} \delta(x-y) \delta\left(v_{x}-v_{y}\right),
\end{aligned}
$$

and

$$
\int_{\mathcal{D}} d x d v\left[\mathcal{L}_{\mathrm{FP}}^{\dagger}\left(x, v_{x}\right) \tau_{\mathcal{D}}\left(x, v_{x}\right)\right] g\left(x, v_{x} \mid y, v_{y}\right)=k \tau_{\mathcal{D}\left(y, v_{y}\right)} .
$$

Hence, the MFPT becomes

$$
\tau_{\mathcal{D}}\left(y, v_{y}\right)=\frac{\int_{\mathcal{D}} d x d v g\left(x, v_{x} \mid y, v_{y}\right)}{\int_{\partial \mathcal{D}} d S_{i} J_{i}\left(x, v_{x} \mid y, v_{y}\right)}
$$

which is precisely the inverse of the flux-over-population formula for the escape rate (2.12), with a source located at $y$ inside the well. 
The escape rate derived with the flux-over-population method is formally equivalent to the inverse of the MFPT. The latter provides a simple interpretation of the escape problem. The escape time, given by the inverse of the escape rate, is similar to the average time needed for a particle to leave a domain. However, the MFPT faces some practical difficulties when solving for the rate, in particular, beyond the overdamped limit. The flux-overpopulation method is better suited to obtain an analytical solution.
[1] H. A. Kramers, Brownian motion in a field of force and the diffusion model of chemical reactions, Physica 7, 284 (1940).

[2] A. Berera, Warm Inflation, Phys. Rev. Lett. 75, 3218 (1995).

[3] A. Berera, Warm inflation at arbitrary adiabaticity: A model, an existence proof for inflationary dynamics in quantum field theory, Nucl. Phys. B585, 666 (2000).

[4] S. Bartrum, A. Berera, and J. G. Rosa, Fluctuation-dissipation dynamics of cosmological scalar fields, Phys. Rev. D 91, 083540 (2015).

[5] L. Farkas, Keimbildungsgeschwindigkeit in übersättigten dämpfen, Z. Phys. Chem. 125, 236 (1927).

[6] P. Talkner, Mean first passage time and the lifetime of a metastable state, Z. Phys. B 68, 201 (1987).

[7] H. Risken and T. Frank, The Fokker-Planck Equation: Methods of Solution and Applications (Springer-Verlag, Berlin, Germany, 1996).

[8] P. Hanggi, P. Talkner, and M. Borkovec, Reaction-rate theory: Fifty years after Kramers, Rev. Mod. Phys. 62, 251 (1990).

[9] P. Reimann, G. J. Schmid, and P. Hänggi, Universal equivalence of mean first-passage time and Kramers rate, Phys. Rev. E 60, R1 (1999).

[10] S. R. Coleman, The fate of the false vacuum. 1. Semiclassical theory, Phys. Rev. D 15, 2929 (1977); Erratum Phys. Rev. D 16, 1248 (1977).

[11] C. G. Callan, Jr. and S. R. Coleman, The fate of the false vacuum. 2. First quantum corrections, Phys. Rev. D 16, 1762 (1977).

[12] A. D. Linde, Decay of the false vacuum at finite temperature, Nucl. Phys. B216, 421 (1983); Erratum Nucl. Phys. B223, 544 (1983).

[13] L. P. Csernai and J. I. Kapusta, Nucleation of relativistic first order phase transitions, Phys. Rev. D 46, 1379 (1992).

[14] S. R. Coleman and F. De Luccia, Gravitational effects on and of vacuum decay, Phys. Rev. D 21, 3305 (1980).

[15] G. Parisi, Statistical Field Theory (Addison-Wesley, Redwood City, USA, 1988).

[16] J. Zinn-Justin, Quantum Field Theory and Critical Phenomena (Oxford/Clarendon Press, Oxford, UK, 2002).

[17] J. S. Langer, Theory of the condensation point, Ann. Phys. (N.Y.) 41, 108 (1967); 281, 941 (2000).

[18] J. S. Langer, Statistical theory of the decay of metastable states, Ann. Phys. (N.Y.) 54, 258 (1969).

[19] S. W. Hawking and I. G. Moss, Supercooled phase transitions in the very early Universe, Phys. Lett. 110B, 35 (1982).
[20] E. A. Calzetta and B. L. B. Hu, Nonequilibrium Quantum Field Theory (Cambridge University Press, Cambridge, England, 2008).

[21] K. c. Chou, Z. b. Su, B. 1. Hao, and L. Yu, Equilibrium and nonequilibrium formalisms made unified, Phys. Rep. 118, 1 (1985).

[22] M. Morikawa, Classical fluctuations in dissipative quantum systems, Phys. Rev. D 33, 3607 (1986).

[23] B. L. Hu, J. P. Paz, and Y. Zhang, in The Origin of Structure in the Universe, edited by E. Gunzig and P. Nardone (Kluwer Acad. Publ., Heidelberg, 1993); E. Calzetta and B. L. Hu, Noise and fluctuations in semiclassical gravity, Phys. Rev. D 49, 6636 (1994); 52, 6770 (1995); B. L. Hu and A. Matacz, Back reaction in semiclassical cosmology: The Einstein-Langevin equation, Phys. Rev. D 51, 1577 (1995).

[24] M. Gleiser and R. O. Ramos, Microphysical approach to nonequilibrium dynamics of quantum fields, Phys. Rev. D 50, 2441 (1994).

[25] A. Berera, M. Gleiser, and R. O. Ramos, Strong dissipative behavior in quantum field theory, Phys. Rev. D 58, 123508 (1998).

[26] A. Berera, I. G. Moss, and R. O. Ramos, Local approximations for effective scalar field equations of motion, Phys. Rev. D 76, 083520 (2007).

[27] A. D. Linde, Particle physics and inflationary cosmology, in Collection: Contemporary Concepts in Physics (Harwood, Chur, 1990).

[28] S. R. Coleman, Quantum tunneling and negative eigenvalues, Nucl. Phys. B298, 178 (1988).

[29] J. S. Langer and L. A. Turski, Hydrodynamic model of the condensation of a vapor near its critical point, Phys. Rev. A 8, 3230 (1973).

[30] G. F. Bonini, A. G. Cohen, C. Rebbi, and V. A. Rubakov, Tunneling of bound systems at finite energies: Complex paths through potential barriers, arXiv:quant-ph/9901062.

[31] J. Ankerhold, Quantum Tunneling in Complex Systems (Springer-Verlag, Berlin, Germany, 2007).

[32] C. M. Bender and D. W. Hook, Quantum tunneling as a classical anomaly, J. Phys. A 44, 372001 (2011).

[33] A. G. Anderson, C. M. Bender, and U. I. Morone, Periodic orbits for classical particles having complex energy, Phys. Lett. A 375, 3399 (2011).

[34] H. Harada, A. Mouchet, and A. Shudo, Riemann surfaces of complex classical trajectories and tunnelling splitting in onedimensional systems, J. Phys. A 50, 435204 (2017).

[35] S. Coleman, Aspects of Symmetry: Selected Erice Lectures (Cambridge University Press, Cambridge, England, 1985). 
[36] R. Venugopalan and A. P. Vischer, Dynamical growth rate of a diffuse interface in first order phase transitions, Phys. Rev. E 49, 5849 (1994).

[37] L. P. Csernai, J. I. Kapusta, and E. Osnes, Domain wall dynamics of phase interfaces, Phys. Rev. D 67, 045003 (2003).

[38] G. D. Moore and K. Rummukainen, Electroweak bubble nucleation, nonperturbatively, Phys. Rev. D 63, 045002 (2001).

[39] M. Gleiser, G. C. Marques, and R. O. Ramos, On the evaluation of thermal corrections to false vacuum decay rates, Phys. Rev. D 48, 1571 (1993).

[40] G. V. Dunne, Functional determinants in quantum field theory, J. Phys. A 41, 304006 (2008).

[41] A. Andreassen, W. Frost, and M. D. Schwartz, Scale invariant instantons and the complete lifetime of the Standard Model, Phys. Rev. D 97, 056006 (2018).

[42] T. W. B. Kibble, Topology of cosmic domains and strings, J. Phys. A 9, 1387 (1976).

[43] A. Vilenkin and E. P. S. Shellard, Cosmic Strings and Other Topological Defects (Cambridge University Press, Cambridge, England, 2000).

[44] T. Vachaspati and M. Barriola, A New Class of Defects, Phys. Rev. Lett. 69, 1867 (1992).

[45] X. Zhang, T. Huang, and R. H. Brandenberger, Pion and eta strings, Phys. Rev. D 58, 027702 (1998).

[46] T. Vachaspati, Vortex Solutions in the Weinberg-Salam Model, Phys. Rev. Lett. 68, 1977 (1992); Erratum Phys. Rev. Lett. 69, 216 (1992).

[47] T. Vachaspati, Electroweak strings, Nucl. Phys. B397, 648 (1993).

[48] A. Berera, R. Brandenberger, J. Mabillard, and R. O. Ramos, Stability of the pion string in a thermal and dense medium, Phys. Rev. D 94, 065043 (2016).
[49] M. R. Douglas, The statistics of string/M theory vacua, J. High Energy Phys. 05 (2003) 046.

[50] L. Susskind, The anthropic landscape of string theory, in Universe or Multiverse?, edited by B. Carr (Cambridge University Press, Cambridge, 2007), pp. 247-266.

[51] J. J. Atick and E. Witten, The Hagedorn transition and the number of degrees of freedom of string theory, Nucl. Phys. B310, 291 (1988).

[52] M. J. Bowick and S. B. Giddings, High temperature strings, Nucl. Phys. B325, 631 (1989).

[53] L. Berthier, G. Biroli, J.-P. Bouchaud, L. Cipelletti, and W. Saarloos, Dynamical Heterogeneities in Glasses, Colloids, and Granular Media (Oxford University Press, Oxford, UK, 2011).

[54] F. H. Stillinger, A topographic view of supercooled liquids and glass formation, Science 267, 1935 (1995).

[55] P. G. Debenedetti and F.H. Stillinger, Supercooled liquids and the glass transition, Nature (London) 410, 259 (2001).

[56] A. A. Starobinsky, Dynamics of phase transition in the new inflationary Universe scenario and generation of perturbations, Phys. Lett. 117B, 175 (1982).

[57] A. A. Starobinsky, Stochastic de Sitter (inflationary) stage in the early Universe, Lect. Notes Phys. 246, 107 (1986).

[58] V. Vennin and A. A. Starobinsky, Correlation functions in stochastic inflation, Eur. Phys. J. C 75, 413 (2015).

[59] E. Nelson, Derivation of the Schrodinger equation from Newtonian mechanics, Phys. Rev. 150, 1079 (1966).

[60] G. Parisi and Y.s. Wu, Perturbation theory without gauge fixing, Sci. Sin. 24, 483 (1981).

[61] P. H. Damgaard and H. Huffel, Stochastic quantization, Phys. Rep. 152, 227 (1987). 